\title{
Statistically Segregated k-Space Sampling for Accelerating Multiple-Acquisition MRI
}

\author{
L. Kerem Senel ${ }^{\circledR}$, Toygan Kilic, Alper Gungor, Emre Kopanoglu ${ }^{\circledR}$, H. Emre Guven, Member, IEEE, \\ Emine U. Saritas ${ }^{\circledR}$, Member, IEEE, Aykut Koc ${ }^{\circledR}$, Member, IEEE, \\ and Tolga Çukur ${ }^{\circledR}$, Senior Member, IEEE
}

\begin{abstract}
A central limitation of multiple-acquisition magnetic resonance imaging (MRI) is the degradation in scan efficiency as the number of distinct datasets grows. Sparse recovery techniques can alleviate this limitation via randomly undersampled acquisitions. A frequent sampling strategy is to prescribe for each acquisition a different random pattern drawn from a common sampling density. However, naive random patterns often contain gaps or clusters across the acquisition dimension that, in turn, can degrade reconstruction quality or reduce scan efficiency. To address this problem, a statistically segregated sampling method is proposed for multiple-acquisition MRI. This method generates multiple patterns sequentially while adaptively modifying the sampling density to minimize k-space overlap across patterns. As a result, it improves incoherence across acquisitions while still maintaining similar sampling density across the radial dimension of k-space. Comprehensive simulations and in vivo results are presented for phase-cycled balanced steady-state free precession and multi-echo $\mathrm{T}_{2}$-weighted imaging. Segregated sampling achieves significantly improved quality in both Fourier and compressed-sensing reconstructions of multiple-acquisition datasets.
\end{abstract}

Manuscript received October 23, 2018; revised December 28, 2018; accepted January 1, 2019. Date of publication January 14, 2019; date of current version June 28,2019 . This work was supported in part by TUBITAK 1001 under Grant 117E171, in part by a European Molecular Biology Organization Installation Grant IG 3028, in part by a TUBA GEBIP Fellowship, and in part by the BAGEP 2016 and BAGEP 2017 Awards of the Science Academy. (Corresponding author: Tolga Cukur.)

L. K. Senel is with the Department of Electrical and Electronics Engineering, Bilkent University, 06800 Ankara, Turkey, also with the National Magnetic Resonance Research Center (UMRAM), Bilkent University, 06800 Ankara, Turkey, And also with the ASELSAN Research Center, 06370 Ankara, Turkey.

T. Kilic is with the Department of Electrical and Electronics Engineering, Bilkent University, 06800 Ankara, Turkey, and also with the National Magnetic Resonance Research Center (UMRAM), Bilkent University, 06800 Ankara, Turkey.

A. Gungor, H. E. Guven, and A. Koc are with the ASELSAN Research Center, 06370 Ankara, Turkey.

E. Kopanoglu is with the ASELSAN Research Center, 06370 Ankara, Turkey, and also with the Cardiff University Brain Research Imaging Centre, School of Psychology, Cardiff University, Cardiff CF24 4HQ, U.K.

E. U. Saritas and T. Çukur are with the Department of Electrical and Electronics Engineering, Bilkent University, Bilkent, 06800 Ankara, Turkey, also with the National Magnetic Resonance Research Center (UMRAM), Bilkent University, Bilkent, 06800 Ankara, Turkey, and also with the Neuroscience Program, Sabuncu Brain Research Center, Bilkent University, Bilkent, 06800 Ankara, Turkey (e-mail: cukur@ee.bilkent.edu.tr).

This paper has supplementary downloadable material available at http://ieeexplore.ieee.org, provided by the author.

Color versions of one or more of the figures in this paper are available online at http://ieeexplore.ieee.org.

Digital Object Identifier 10.1109/TMI.2019.2892378
Index Terms-Sampling pattern, incoherence, k-space coverage, variable density, multiple acquisition, compressed sensing.

\section{INTRODUCTION}

$\mathbf{M}$ ULTIPLE-ACQUISITION MRI methods are used when the image quality or information content of a single acquisition is insufficient. These methods acquire multiple images of the same anatomy, typically with different sequence parameters and image contrasts. Examples include phase-cycled balanced steady-state free precession (bSSFP) and multi-echo $\mathrm{T}_{2}$-weighted imaging. Typical uses of multiple acquisitions include improved suppression of background tissues [1], [2], relaxometry [3], extended slice coverage [4], separation of distinct resonances [5], [6], and reduction of image artifacts [7], [8]. While performance scales with the number of datasets acquired $(\mathrm{N})$, this results in longer scan times and increased motion sensitivity. Therefore, multipleacquisition methods can greatly benefit from acceleration techniques that enable high scan efficiency.

Leveraging the sparse nature of MR images, compressed sensing (CS) techniques [9]-[12] were recently proposed to accelerate multiple-acquisition MRI. This powerful approach was demonstrated in several applications including fat-water separation [13]-[15], parametric mapping [16]-[20], diffusionweighting imaging [21]-[23], subtraction angiography [24], multi-contrast imaging [25]-[27], and lately bSSFP imaging [28]. Individual acquisitions were accelerated via variabledensity sampling patterns because the energy spectrum of MRI images is heavily constrained to central k-space [9], [29]. Unacquired k-space data were then recovered via nonlinear reconstructions that enforce compressibility in a transform domain [30]-[32].

The success of CS reconstructions depends critically on the selection of k-space sampling locations. Much work has been done on optimizing sampling patterns for single-acquisition MRI. Theory indicates that random patterns that promote incoherent aliasing guarantee sparse recovery with high probability [33], [34]. Thus, many early studies proposed variabledensity random patterns to maximize incoherence of aliasing artifacts in spatial or temporal dimensions [9], [12], [35], [36]. Improved strategies were later considered to maintain a favorable compromise between incoherence and practical imaging considerations. For instance, pattern formation based 
on adaptive density estimation was suggested to effectively utilize prior information about the energy spectrum of specific datasets [37]-[39]. Recent studies also imposed deterministic constraints on sampling patterns to prevent unwanted gaps or clusters. Examples of this approach include Poisson disc sampling to improve uniformity of inter-sample distances in multi-coil imaging [40], optimization routines to maintain fixed frame rates in dynamic imaging [41], [42], complementary Poisson sampling for variable view sharing in dynamic contrast-enhanced imaging [43], and sample ordering to minimize eddy-current artifacts in segmented acquisitions [44]. Hybrid strategies were also proposed that deploy deterministic sampling in central and random sampling in peripheral regions of k-space to better suppress aliasing artifacts in reconstructed images [45].

Contrary to single-acquisition MRI, less attention has been given to sampling strategies for multiple static acquisitions. As such, the standard practice in the field is to accelerate each acquisition via a different random pattern drawn from a fixed sampling density [14], [25], [27]. Because separate instances of a random variable are independent, this strategy is theoretically expected to yield incoherent aliasing across acquisitions, which should then translate to successful recovery in CS [9]. Yet, naive random selection can leave gaps/clusters in the acquisition dimension that can degrade reconstruction quality or reduce scan efficiency. Improved sampling methods previously proposed for single-acquisition MRI commonly use deterministic contstraints that limit the randomness of the resulting patterns [41]-[44]. While a recent method for multiple-acquisition MRI selects from random patterns to minimize inter-pattern correlations [28], it is computationally intensive and suboptimal in minimizing pattern overlap across acquisitions.

Here we aim to minimize k-space overlap across patterns for separate acquisitions, while maintaining randomness and similar sampling density in individual patterns. To achieve this goal, we devise a mathematical framework for statisticallysegregated sampling in multiple-acquisition MRI. The proposed method generates each of $\mathrm{N}$ patterns in sequence, while adaptively modifying the sampling density to promote minimal pattern overlap. For each pattern, the sampling probability is lowered for $\mathrm{k}$-space locations that are covered by preceding patterns. The probability for uncovered locations is appropriately increased to maintain identical sampling density across radial k-space within each pattern. Segregated sampling preserves the stochastic nature of individual patterns while increasing $\mathrm{k}$-space coverage. It significantly reduces pattern overlap compared to random sampling, without the need for time-consuming optimization procedures. Simulations and in vivo results on phase-cycled bSSFP and multi-contrast imaging clearly demonstrate improved quality in Fourier and CS reconstructions of multiple-acquisition datasets.

\section{A. Multiple-Acquisition MRI}

\section{THEORY}

Here we consider two multiple-acquisition applications, phase-cycled bSSFP imaging and multi-contrast $\mathrm{T}_{2}$-weighted imaging. Main field inhomogeneities can introduce regions of signal voids in bSSFP images known as banding artifacts [7]. To prevent signal loss, phase-cycled bSSFP methods acquire multiple images of the same anatomy with nearly identical contrast except for a spatial shift in the location of artifacts [46]. This is implemented by applying a unique phasecycling value between consecutive RF pulses during each acquisition. The resulting bSSFP signal can be expressed as,

$$
S_{n}(\vec{r})=M(\vec{r}) \frac{e^{i\left(\phi(\vec{r})+\Delta \phi_{n}\right) / 2}\left(1-A(\vec{r}) e^{-i\left(\phi(\vec{r})+\Delta \phi_{n}\right)}\right)}{1-B(\vec{r}) \cos \left(\phi(\vec{r})+\Delta \phi_{n}\right)}
$$

under the assumption that the echo time (TE) is half the repetition time (TR). In Eq. (1), $\vec{r}$ denotes the spatial location vector, $\phi$ is the phase accrued in a TR due to field inhomogeneity, and $\Delta \phi_{n}$ is the phase-cycling value selected for the $\mathrm{n}^{\text {th }}$ acquisition $\left(\mathrm{n} \in\left[\begin{array}{ll}1 \mathrm{~N} & \mathrm{~N}\end{array}\right) . M, A, B\right.$ that do not depend on field inhomogeneity are described elsewhere [28]. Multiple bSSFP acquisitions with differential sensitivity to field inhomogeneity carry similar information about tissue structure. These acquisitions can be simply combined [8], [47] or jointly reconstructed [48] to suppress banding artifacts.

Spin-echo ( $\mathrm{SE}$ ) imaging with $\mathrm{T}_{2}$-weighted contrast is pervasive in anatomical assessment. However, a single $\mathrm{T}_{2}$-weighting may be suboptimal when relaxation parameters vary substantially across subjects [49] or when tissues show relatively broad variation in $\mathrm{T}_{2}$ values. In such cases, multiple $\mathrm{T}_{2}$-weighted images with varying $\mathrm{TE}$ values can be colllected. The resulting SE signal is [50],

$$
S_{n}(\vec{r})=i M(\vec{r}) \cdot\left(1-e^{-T R / T_{1}(\vec{r})}\right) \cdot\left(e^{-T E_{n} / T_{2}(\vec{r})}\right)
$$

under the assumption that $\mathrm{TR} \gg \mathrm{TE}_{n}$. In the above equation, $\mathrm{T}_{1}(\vec{r})$ and $\mathrm{T}_{2}(\vec{r})$ denote the spatial distribution of longitudinal recovery and transverse relaxation time constants, respectively. $\mathrm{TE}_{n}$ denotes the echo-time of the respective $\mathrm{SE}$ acquisition where $\mathrm{n} \in\left[\begin{array}{ll}1 \mathrm{~N}\end{array}\right]$. Because multiple $\mathrm{SE}$ acquisitions with differential $\mathrm{T}_{2}$-weighting as in Eq. (2) carry shared tissue information (e.g., location of tissue boundaries), they can be jointly reconstructed [25], [26] to improve image quality and to enhance tissue discrimination [51].

Prescribing a larger $\mathrm{N}$ significantly improves image quality in both bSSFP and multi-contrast applications. Meanwhile, undesirable lengthening of scan times can be prevented through k-space undersampling. The unacquired data can then be estimated by solving an inverse problem based on the following forward model:

$$
y_{n}(\vec{k})=D_{n} \mathcal{F}\left\{S_{n}(\vec{r})\right\}
$$

Here $y_{n}$ denotes the $\mathrm{k}$-space data for the $\mathrm{n}^{\text {th }}$ acquisition, $\vec{k}$ is the k-space location vector, $\mathcal{F}$ is the Fourier-transformation, and $D_{n}$ is a binary mask that reflects the $n^{\text {th }}$ sampling pattern.

\section{B. Variable-Density Random Sampling}

The energy spectrum of MRI images follow an approximate power-law in k-space [29]. The transform domain coefficients also tend to be sparser at fine-scales that reflect high spatial frequencies [9]. As a result, variable-density random sampling (VDS) has come forth as a preferred companion to CS reconstructions. In VDS, the expected sampling density 
function (PDF) is specified to maintain a desired acceleration rate. For multiple-acquisition MRI, the sampling PDF is usually taken to be identical across acquisitions:

$$
p_{D_{n}}\left(k_{y}, k_{z}\right)=p_{o}\left(k_{r}\right)
$$

where $k_{r}=\sqrt{k_{y}^{2}+k_{z}^{2}}$ is the $\mathrm{k}$-space radius, $p_{o}$ is the common density, and circular symmetry is assumed across phase-encoding dimensions without loss of generality. This density is then used to draw random instances of sampling patterns for each acquisition, i.e.,

$$
p_{D_{n}}\left(k_{y}, k_{z}\right) \stackrel{\text { draw }}{\longrightarrow} D_{n}\left(k_{y}, k_{z}\right)
$$

Although random sampling often yields a high degree of incoherence, the generated patterns may occasionally have poor aliasing properties. As a remedy, Monte-Carlo designs have been proposed where multiple sets of candidate patterns are drawn, $D_{n}^{c}\left(k_{y}, k_{z}\right)$ [9]. The incoherence of each pattern is measured via its point spread function (PSF). An image containing a unit-intensity voxel is undersampled in k-space with the given pattern, and re-transformed to the image domain to calculate the PSF. The ratio of peak intensity to maximum side-lobe intensity of the PSF $\left(R_{P S F}\right)$ reflects incoherence. In this random sampling method, the candidate pattern with the maximum $R_{P S F}$ is selected for each acquisition independently:

$$
D_{n}=\max _{D_{n}^{c}} R_{P S F}\left(D_{n}^{c}\right)
$$

Other desired properties can also be enforced, such as minimal correlation among patterns for multiple acquisitions [28]. In this low-correlation method, a large number of candidates are first generated for the set of multiple patterns across acquisitions. The set of patterns with minimum pair-wise correlations can then be identified through brute-force search:

$$
\left\{D_{1}, \ldots, D_{N}\right\}=\min _{D_{1, . ., N}^{c}}\left(\sum_{i=1}^{N} \sum_{j=i+1}^{N} \operatorname{corr}\left(D_{i}^{c}, D_{j}^{c}\right)\right)
$$

\section{Sampling Performance: Coverage and Overlap}

Given their stochastic nature, random patterns are best analyzed in a statistical framework. In random sampling, patterns are generated independently and the inclusion of a k-space location within each pattern follows a Bernoulli distribution (with parameter $p_{o}\left(k_{r}\right)$ ). Thus, the total number of times ( $\mathrm{t}$ ) a $\mathrm{k}$-space location is sampled across $\mathrm{N}$ acquisitions follows a binomial distribution:

$$
P_{t}\left(k_{y}, k_{z}\right)=\left(\begin{array}{c}
N \\
t
\end{array}\right) p_{o}\left(k_{r}\right)^{t}\left[1-p_{o}\left(k_{r}\right)\right]^{(N-t)}
$$

The probability of complete omission from all acquisitions is:

$$
P_{0}\left(k_{y}, k_{z}\right)=\left(1-p_{o}\left(k_{r}\right)\right)^{N}
$$

The probability of being sampled within a single acquisition is:

$$
P_{1}\left(k_{y}, k_{z}\right)=N\left(1-p_{o}\left(k_{r}\right)\right)^{N-1} p_{o}\left(k_{r}\right)
$$

Here we give attention to three properties of multipleacquisition patterns: aggregate coverage, differential coverage, and overlap. We take the aggregate coverage of $\mathrm{N}$ patterns as the proportion of k-space locations that are sampled in at least one pattern:

$$
\% \text { coverage }=\frac{1}{T} \sum_{k_{y}, k_{z}}\left(1-P_{0}\left(k_{y}, k_{z}\right)\right)
$$

where $T$ denotes the total number locations in the sampling grid. Meanwhile, we take differential coverage as the proportion of locations that are uniquely sampled within an individual pattern:

$$
\% \text { differential cov. }=\frac{1}{T} \sum_{k_{y}, k_{z}}\left(P_{1}\left(k_{y}, k_{z}\right)\right)
$$

Lastly, we take overlap among patterns as the number of times a location has been repeatedly sampled across acquisitions:

$$
O= \begin{cases}t-1, & \text { if } t \geqslant 2 \\ 0, & \text { otherwise }\end{cases}
$$

The percentage overlap is then measured as the expected value of $O$ averaged across k-space:

$$
\% \text { overlap }=\frac{1}{T .(N-1)} \sum_{k_{y}, k_{z}} \sum_{t=2}^{N}(t-1) \cdot P_{t}\left(k_{y}, k_{z}\right)
$$

Eq. 11 clearly shows that aggregate coverage decreases towards high spatial frequencies (i.e., lower sampling density) and with smaller $\mathrm{N}$. If aggregate coverage is broadened by increasing either the sampling density or $\mathrm{N}$, the range of achievable acceleration factors will be limited and the total scan time will be prolonged. Note that higher sampling density and larger $\mathrm{N}$ will result in decreased differential coverage (Eq. 12) and increased pattern overlap (Eq. 14). As a result, redundant or highly similar information will be collected across acquisitions, reducing scan efficiency. This inherent trade-off poses a significant limitation on the utility of random sampling.

\section{Statistically Segregated k-Space Sampling}

Here we propose a statistically-segregated sampling method that broadens the aggregate coverage of multiple patterns to increase the amount of tissue information captured. In random sampling, major increases in $p_{o}\left(k_{r}\right)$ or $\mathrm{N}$ are required to boost coverage, but these changes prolong scan times. In segregated sampling, coverage is enhanced by controlling for unwanted overlap across patterns while retaining the same $\mathrm{N}$ and radial sampling density.

The proposed method is implemented via a statistical framework (Fig. 1) where the joint probability distribution for $\mathrm{N}$ patterns is:

$$
\begin{aligned}
p_{D_{1}, . ., D_{N}}\left(k_{y 1, z 1}, . ., k_{y N, z N}\right) & =p_{D_{N} \mid \bar{D}_{N-1}} \cdot . . \cdot p_{D_{2} \mid \bar{D}_{1}} \cdot p_{D_{1}} \\
\text { subj. to } p_{D_{n} \mid \bar{D}_{n-1}}\left(k_{r}\right) & =\int_{k_{\theta}} p_{D_{n} \mid \bar{D}_{n-1}}\left(k_{r}, k_{\theta}\right)=p_{o}\left(k_{r}\right)
\end{aligned}
$$




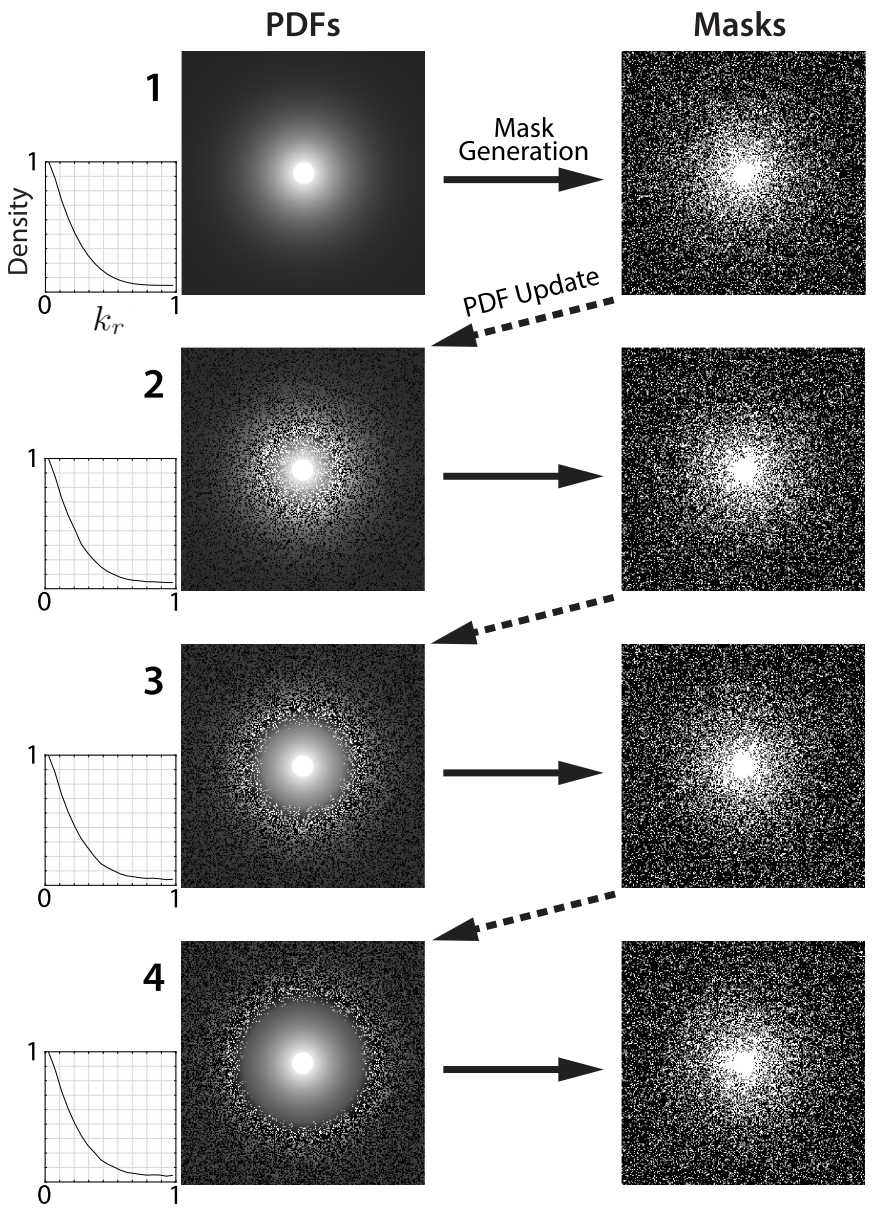

Fig. 1. Segregated sampling designs $\mathrm{N}$ random undersampling patterns (Masks) via Monte-Carlo simulations based on respective sampling density functions (PDFs). Unlike standard random sampling, it adaptively modifies the sampling density to increase aggregate k-space coverage and to promote minimal pattern overlap $(\mu=0)$. It lowers sampling density in k-space locations that are readily covered in preceding patterns, and increases sampling density for uncovered locations. At a given $\mathrm{k}$-space radius $\left(k_{r}\right)$, the total increase in density for uncovered locations is equal to the total decrease for covered locations. This procedure yields incoherent patterns across the acquisition dimension while maintaining identical sampling density across $k_{r}$.

where $\bar{D}_{n}=\left\{D_{n}, . . D_{1}\right\}$. The joint distribution is decomposed into conditional distributions, constrained to follow a preselected sampling density $\left(p_{o}\right)$ across radial k-space $\left(k_{r}\right)$. The conditional distributions and respective sampling patterns are generated sequentially, starting with $D_{1}$ :

$$
p_{D_{1}}\left(k_{y, z}\right)=p_{o}\left(k_{r}\right)
$$

Overlap in subsequent patterns is minimized by decreasing the sampling density $\left(p^{-}\right)$in previously covered locations while increasing it $\left(p^{+}\right)$in uncovered locations:

$$
\begin{aligned}
& p_{D_{n} \mid \bar{D}_{n-1}}^{-}\left(k_{y, z}^{-}\right)=p_{o}\left(k_{r}\right) \cdot \mu \\
& p_{D_{n} \mid \bar{D}_{n-1}}^{+}\left(k_{y, z}^{+}\right)=p_{o}\left(k_{r}\right) \cdot \beta_{n-1}\left(k_{r}\right)
\end{aligned}
$$

where $k_{y, z}^{-}$denotes locations covered at least once in previous patterns (i.e., $\sum_{i=1}^{n-1} D_{i} \geqslant 1$ ), and $k_{y, z}^{+}$denotes the remaining uncovered locations. Note that the above density modifications are only viable at $k_{r}$ values where the set of uncovered locations is non-empty. The modification is then controlled via the parameter $\mu \in\left[\begin{array}{l}01 \\ 1\end{array}\right.$, which results in random sampling at $\mu=1$ and maximally segregated sampling at $\mu=0$. Meanwhile, the parameter $\beta_{n}$ is dependent on $\mu$ :

$$
\beta_{n}\left(k_{r}\right)=\frac{1-\mu \cdot K_{n}^{-}\left(k_{r}\right)}{1-K_{n}^{-}\left(k_{r}\right)}
$$

For a given $k_{r}, K_{n}^{-}$denotes the ratio of the number of unique locations sampled in patterns $\left\{D_{1}, . . D_{n}\right\}$ to the number of locations on the sampling grid, where $K_{n}^{-}$is estimated for each $n$. This $\beta_{n}$ definition ensures that a fixed radial sampling density -in accordance with $p_{o}\left(k_{r}\right)$ - is maintained (see Fig. 1 for example). Note that the omission of $\beta_{n}$ in Eq. 18 would lower the average sampling density at $k_{r}$, and thereby lead to sampling patterns with fewer samples than dictated by the desired acceleration factor.

The proportion of grid locations sampled, $K_{n}^{-}$, grows steadily with $n$, and the growth rate depends on $p_{o}$ and varies across $k_{r}$. This rate can be examined by calculating its expected value $e_{n}\left(k_{r}\right)=E\left\{K_{n}^{-}\left(k_{r}\right)\right\}$. As expected $e_{1}=p_{o}$, and for subsequent acquisitions:

$$
\begin{aligned}
e_{n} & =e_{n-1}+\left(1-e_{n-1}\right) E\left\{p_{D_{n} \mid \bar{D}_{n-1}}^{+} \mid K_{n-1}^{-}\right\} \\
& =e_{n-1}+\left(1-e_{n-1}\right) \cdot\left(p_{o} \frac{1-\mu e_{n-1}}{1-e_{n-1}}\right) \\
& =e_{n-1}\left(1-\mu p_{o}\right)+p_{o}
\end{aligned}
$$

The solution of the difference equation in Eq. 20 is:

$$
e_{n}=\frac{1}{\mu}-\frac{1}{\mu}\left(1-\mu p_{o}\right)^{n}
$$

Note that as $n$ takes on increasingly greater values, $e_{n}$ in the above solution converges to $1 / \mu$ in the limit. Therefore, it is possible to have $e_{n-1}<1$ and $e_{n} \geq 1$ for a finite value of $n$ (equivalently $K_{n-1}^{-}<1$ and $K_{n}^{-}=1$ ). However, $e_{n} \geq 1$ suggests that the modified probability values $p_{D_{n} \mid \bar{D}_{n-1}}^{+}$ exceed 1, indicating that further segregation is not possible. Eq. 21 is no longer valid when this violation is detected, since a corrected set of rules must be used for density modification instead of Eqs. 17 and 18:

$$
\begin{aligned}
& p_{D_{n} \mid \bar{D}_{n-1}}^{-}\left(k_{y, z}^{-}\right)=\frac{K_{n}^{-}\left(k_{r}\right)-1+p_{o}\left(k_{r}\right)}{K_{n}^{-}\left(k_{r}\right)} \\
& p_{D_{n} \mid \bar{D}_{n-1}}^{+}\left(k_{y, z}^{+}\right)=1
\end{aligned}
$$

This updated rule ensures that the maximum density value is 1 , and the density for the remaining locations is adjusted to maintain $p_{o}\left(k_{r}\right)$ in the radial dimension. Once $K_{n}^{-}\left(k_{r}\right)=1$ is reached, all k-space locations at $k_{r}$ would have been sampled at least once. Thus subsequent patterns are drawn from the original density $p^{-}=p_{o}\left(k_{r}\right)$.

\section{Methods}

\section{A. Generation of Sampling Patterns}

Random and segregated sampling patterns were generated. A common PDF was designed to achieve a target acceleration factor (R) isotropically in the two phase-encode dimensions. 
For variable density sampling, the PDF was designed based on a polynomial function of k-space radius [9]; the polynomial degree monotonically increased with $\mathrm{R}$ : degrees were $(2,3,4$, $5,6)$ for $\mathrm{R}=(2,3,4,6,8)$. For uniform density sampling, a PDF with constant sampling probability was used. Patterns were drawn from the designed PDFs via a Monte Carlo procedure described previously [36]. For random and segregated sampling, the pattern that minimized aliasing energy was selected among 1000 candidate instances.

For comparison, Poisson-disc sampling patterns were also generated since they are known to increase sampling uniformity in k-space. Similar to random and segregated sampling, patterns were designed to achieve isotropic acceleration in the two phase-encode dimensions. A distinct Poisson-disc pattern was drawn for each acquisition. Random patterns based on variable-density and Poisson-disc methods were compared in joint reconstructions of in vivo multiple-acquisition data (see Supp. Table I). The variable-density patterns outperformed Poisson-disc patterns in all examined cases, so random and segregated sampling were implemented based on variabledensity patterns for all reconstructions thereafter.

2D CAIPIRINHA sampling patterns [52] were generated for parallel imaging to better distribute aliasing between the two phase-encode dimensions. Among the set of alternative CAPIRINHA patterns, the one that provided the highest reconstruction quality was reported at each R. CAIPIRINHA patterns were also examined for joint reconstructions. Note that CS reconstructions rely on incoherent aliasing in the transform domain. As such, deterministic CAIPIRINHA patterns performed suboptimally (with over $12 \mathrm{~dB}$ performance loss) compared to uniform-density random sampling, so they were not considered thereafter.

Depending on $\mathrm{R}$, a central $\mathrm{k}$-space region reaching $4 \%$ to $18 \%$ of the maximum spatial frequency was sampled at the Nyquist rate for all sampling patterns. For the numerical brain phantom, the sampling grid sizes were $434 \times 362$ in $\mathrm{T}_{1}$-weighted and bSSFP acquisitions, and $362 \times 434$ in $\mathrm{T}_{2}$-weighted acquisitions. For in vivo experiments, the grid sizes were $256 \times 256$ for bSSFP acquisitions and $192 \times 224$ for $\mathrm{T}_{2}$-weighted acquisitions. To more closely match the sizes of the numerical phantom and in vivo images, lower-resolution variants of the phantom were also generated by downsampling the dimensions from 434 to 278 samples and from 362 to 232 samples.

All sampling patterns were generated in MATLAB (MathWorks, MA) using libraries from the SPIRiT toolbox [40]. The segregated sampling algorithm is available at https: / / github.com/icon-lab/mrirecon/ segSampling/.

\section{B. Reconstruction of Multiple-Acquisition Data}

Four different reconstruction methods were comparatively evaluated on multiple-acquisition datasets. First, Fourier reconstructions of individual acquisitions $(\mathrm{ZF})$ were computed: unacquired data were filled with zeros, data were compensated for the variable sampling density across k-space, and lastly an inverse Fourier transformation was taken. Second, a profile-encoding (PE) reconstruction was performed on multiple acquisitions [48]. To minimize computational load and enhance sensitivity to potential effects of segregated sampling, a single-channel complex dataset was obtained for each acquisition via an optimal linear combination across coils [53]. Coil sensitivities were estimated based on the central $16 \times 16$ region of $\mathrm{k}$-space. Multiple-acquisition datasets were then reconstructed using the iterative self-consistent parallel imaging (SPIRiT) method, originally proposed for coil arrays [40]. Third, multiple coil data for each individual acquisition were separately reconstructed using parallel imaging (PI). The conventional GRAPPA method was used for this purpose [54]. Fourth, a joint PE and PI reconstruction was performed on multiple-coil multiple-acquisition data. An implementation of SPIRiT generalized to interpolate across multiple coils and acquisitions was used for this purpose [55]. To reduce computational load, a geometric coil compression (GCC) was performed on the datasets prior to reconstruction that reduced the number of coils from 4 to 2 for bSSFP data and from 32 to [2 16] for $\mathrm{T}_{2}$-weighted data [56].

The PE and joint reconstructions considered here are based on the SPIRiT formalism [40]. SPIRiT aims to express a given sample as a weighted combination of neighboring samples across acquisitions in $\mathrm{PE}$, and across acquisitions and coils in joint reconstructions. An interpolation kernel $\left(\mathcal{K}_{n, d}\right)$ is estimated from calibration data in the central region of $\mathrm{k}$-space to linearly synthesize unacquired data. Reconstructions are performed by solving the following optimization problem:

$$
\begin{aligned}
\min _{\substack{\left\{m_{n, d}\right\}_{n=1 \ldots N} \\
d=1 \ldots N_{c}}} & \sum_{n, d}\left\|y_{n, d}-\mathcal{F}_{\mathcal{P}_{n, d}}\left\{m_{n, d}\right\}\right\|_{2}^{2} \\
& +\sum_{n, d} \lambda_{0}\left\|\left(\mathcal{G}_{n, d}-I\right) m_{n, d}\right\|_{2}^{2}+\lambda_{1}\left\|\sqrt{\sum_{n, d}\left|\psi\left\{m_{n, d}\right\}\right|^{2}}\right\|_{1}
\end{aligned}
$$

where $m_{n, d}$ is the reconstructed image for the $n^{\text {th }}$ acquisition (where $n \in\left[\begin{array}{ll}1 & N\end{array}\right]$ ) and $d^{t h}$ coil (where $d \in\left[\begin{array}{ll}1 & N_{c}\end{array}\right]$ ), and $\mathcal{G}_{n, d}$ is the image-domain equivalent of $\mathcal{K}_{n, d}$. The first term in the objective enforces the consistency of acquired data $\left(y_{n, d}\right)$ with the reconstructed data $\left(\mathcal{F}_{\mathcal{P}_{n, d}}\left\{m_{n, d}\right\}\right)$. The second term enforces the consistency of the interpolation kernel $\left(\mathcal{G}_{n, d}\right)$ with the reconstructed images $\left(m_{n, d}\right)$. The third term is used to enforce joint-sparsity of the reconstructed images in a known transform domain $(\psi)$ [31].

For both numerical phantom and in vivo data, we primarily considered 2D acceleration in 3D Cartesian acquisitions, where the readout dimension is fully sampled. Fourier transformation was performed across the readout dimension, yielding a separate 2D cross section at each spatial location in that dimension. Each cross section spanning across the undersampled phase-encode dimensions was reconstructed independently. Thus, $\mathcal{K}_{n, d}$ was estimated for interpolation in a $11 \times 11 \mathrm{k}$-space neighborhood. Although the fully-sampled $\mathrm{k}$-space radius varied between $4 \%$ to $18 \%$, variable-density patterns effectively sample a broader region approximately at the Nyquist rate. Thus to more effectively use information in acquired data, $\mathcal{K}_{n}$ was trained in a calibration region of size 
$96 \times 96$ (see Supp. Fig. 1 for the effect of different sizes of the calibration region). Note that the calibration region included not only the fully-sampled region in k-space but spanned across the broader densely-sampled region. This definition of the calibration region was observed to improve kernel estimates, without altering scan efficiency or acceleration rate. Tykhonov regularization with weight $\alpha=0.01$ was used during training. Prior to reconstruction, data were normalized to set the norm of density-compensated data divided by the square root of $\mathrm{N}$ to 1 (i.e. $\ell_{2}$ normalization). The operator $\psi$ was a Daubechies 4 wavelet. Eq. 24 was decomposed into two subproblems using variable splitting with a splitting parameter of 1 . The first problem containing the data and calibration consistency terms was solved via a conjugate gradient (CG) algorithm [31]. The second problem containing the sparsity term was solved via soft thresholding. For numerical phantom data, a total of $30 \mathrm{CG}$ iterations and $\lambda_{0}=10^{-6}$, $\lambda_{1}=0,1$ outer iteration were used. For in vivo bSSFP data, $40 \mathrm{CG}$ iterations, $\lambda_{0}=10^{-6}, \lambda_{1}=10^{-3}$, 30 outer iterations were used. For in vivo multi-contrast data, 15 CG iterations, $\lambda_{0}=10^{-6}, \lambda_{1}=5 \times 10^{-3}, 30$ outer iterations were used for all reconstructions except $\lambda_{1}=5 \times 10^{-4}$ during joint reconstructions. All reconstructions were implemented in MATLAB (MathWorks, MA) using libraries from the SPIRiT toolbox [40].

\section{Simulations}

To theoretically assess sampling performance, sampling strategies were compared in terms of their aggregate coverage (Eq. (11)), differential coverage (Eq. (12)) and percentage overlap (Eq. (14)). Random and segregated sampling ( $\mu=0$, maximally segregated) patterns were generated for $\mathrm{N}=\left[\begin{array}{l}216 \\ 16\end{array}\right.$ and $\mathrm{R}=\left[\begin{array}{ll}2 & 8\end{array}\right]$. (Low-correlation sampling masks yielded limited improvement over random sampling, so they were excluded from comparison.) Differential coverage and overlap were normalized by the maximum coverage of a single pattern dictated by R. To examine the effect of k-space size, patterns were generated for 4 distinct k-space grid sizes $64 \times 64$, $128 \times 128,256 \times 256$, and $384 \times 384$.

Sampling performance was assessed on a numerical brain phantom at $0.5 \mathrm{~mm}$ isotropic resolution (http://www.bic.mni.mcgill.ca/brainweb). A single $\mathrm{T}_{1}$ weighted image was simulated based on Eq. 2. Multiple acquisitions were obtained from this image by using $\mathrm{N}$ patterns, each with an undersampling factor of N. Random and segregated $(\mu=0)$ sampling were used to undersample in two phase-encode dimensions. The following $T_{1} / T_{2}$ values were used: $2570 / 330 \mathrm{~ms}$ for cerebro-spinal fluid (CSF), 1200/250 ms for blood, 500/70 ms for white matter, $830 / 83 \mathrm{~ms}$ for gray matter, $970 / 50 \mathrm{~ms}$ for muscle, and 350/70 ms for fat [57]. The parameters of SE acquisitions were $\alpha=90^{\circ}-180^{\circ}$ (excitation and refocusing pulses), $\mathrm{TR}=575 \mathrm{~ms}$, and $\mathrm{TE}=14 \mathrm{~ms}$. ZF reconstructions were summed across acquisitions.

Next, simulations were performed to demonstrate segregated sampling in the presence of variations in image structure across acquisitions. In phase-cycled bSSFP simulations, the signal for each tissue was calculated using Eq. 1. The following $T_{1} / T_{2}$ values were used: $3000 / 1000 \mathrm{~ms}$ for cerebrospinal fluid (CSF), $1200 / 250 \mathrm{~ms}$ for blood, $1000 / 80 \mathrm{~ms}$ for white matter, $1300 / 110 \mathrm{~ms}$ for gray matter, $1400 / 30 \mathrm{~ms}$ for muscle, and $370 / 130 \mathrm{~ms}$ for fat [49]. Meanwhile, the PD values were: 1 for CSF, blood, muscle and fat, 0.77 for white matter, and 0.86 for gray matter. The bSSFP sequence parameters were set to $\alpha=45^{\circ}$ (flip angle), TR/TE $=5.0 / 2.5 \mathrm{~ms}$, and $\Delta \phi$ spanning the range $\left[\begin{array}{ll}0 & 2 \pi\end{array}\right)$ in steps of size $2 \pi / N$ with $N=8$. A main field inhomogeneity map was used that yielded off-resonance shifts of $0 \pm 62 \mathrm{~Hz}$ (mean \pm std across the volume). Balanced SSFP acquisitions were undersampled by a factor of $\mathrm{N}$ in two phase-encode dimensions using random and segregated $(\mu=0)$ sampling. PE reconstructions were performed for $\mathrm{N}=2,4,6,8$ and $\mathrm{R}=\mathrm{N}$. Individual phasecycled images were p-norm combined across acquisitions $(\mathrm{p}=2)$.

Multi-contrast $\mathrm{T}_{2}$-weighted images of the numerical brain phantom were simulated based on Eq. 2. Relaxation parameters were identical to those used in bSSFP simulations. The parameters of SE were $\alpha=90^{\circ}-180^{\circ}$, TR $=2800 \mathrm{~ms}$, and $\mathrm{TE}=(60,100,140) \mathrm{ms}$ corresponding to $\mathrm{N}=3$. PE reconstructions were computed on acquisitions undersampled with $\mathrm{R}=3$ in two phase-encode dimensions using random and segregated $(\mu=0)$ sampling.

The interaction between noise level and sampling performance was examined on bSSFP and $\mathrm{T}_{2}$-weighted images. Independent bivariate Gaussian noise patterns with zero mean and variance ranging from $10^{-6}$ to $10^{-2}$ were added to 10 different cross sections. For bSSFP reconstructions with $N<8$, symmetric subsets of phase-cycles were used except for $N=6$ where a random subset was selected for each cross section. PE reconstructions were performed on noisy data undersampled with random and segregated $(\mu=0)$ patterns. The following parameters were used: $\mathrm{N}=(4,6,8)$ and $\mathrm{R}=$ $\mathrm{N}$ for bSSFP images, and $\mathrm{N}=3$ and $\mathrm{R}=3$ for $\mathrm{T}_{2}$-weighted images.

To examine the effect of k-space coverage on image quality, the parameter $\mu$ in Eq. 17 was tuned to systematically vary aggregate coverage from that of random sampling to that of segregated sampling. Phase-cycled bSSFP images of the brain were undersampled for $\mu=(0,0.2,0.4,0.6,0.8,1.0)$. At each value of $\mu$, PE reconstructions were performed for $\mathrm{N}=4,6,8$ and $\mathrm{R}=\mathrm{N}$. The simulations were repeated for 10 independent sets of sampling patterns.

Reconstruction were compared to Fourier reconstructions of fully-sampled acquisitions. For bSSFP images, comparisons were performed on the combination image across phasecycles. For multi-contrast images, comparisons were performed individually on each contrast image. Each image was normalized by mapping the $\% 98$ percentile of pixel intensity to 1. Peak signal-to-noise ratio (PSNR), structural similarity index (SSIM), and root mean-squared error (RMSE) were then measured. Error and structural similarity maps were generated for visualization. Separate reconstructions were obtained for 10 different cross sections with different instances of sampling patterns. 
a

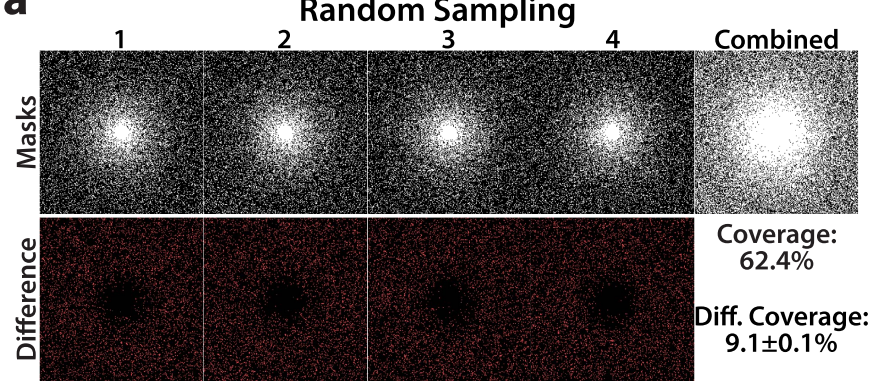

b

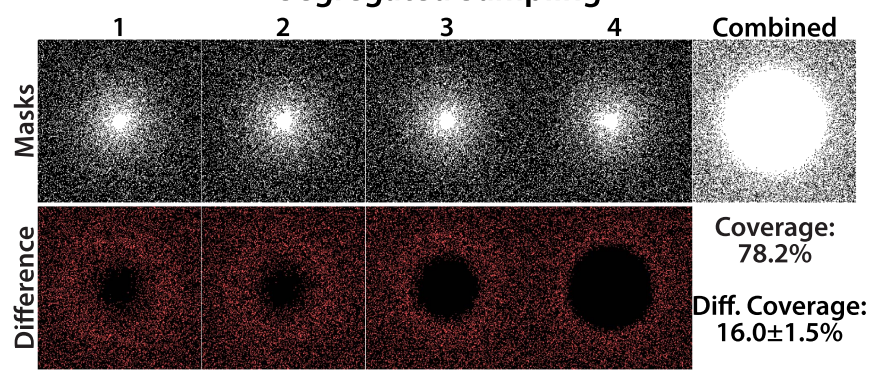

Fig. 2. Representative sampling patterns on a $256 \times 256$ sampling grid for $\mathrm{N}=4, \mathrm{R}=4$ generated using (a) random sampling and (b) segregated sampling $(\mu=0)$. The resulting patterns and the aggregate pattern (Combined) are shown in upper rows. The difference masks comprising locations that are uniquely sampled by each pattern are shown in bottom rows. Segregated sampling increases aggregate k-space coverage to $78.2 \%$ from merely $62.4 \%$ in random sampling. It also increases the average differential coverage $16.0 \pm 1.5 \%$ (mean \pm std across $N$ ) from $9.1 \pm 0.1 \%$ in random sampling, due to reduced pattern overlap.

\section{Experiments}

The sampling strategies were demonstrated in vivo using a $3 \mathrm{~T}$ Siemens scanner (maximum gradient strength of $45 \mathrm{mT} / \mathrm{m}$ and slew rate of $200 \mathrm{~T} / \mathrm{m} / \mathrm{s})$. First, brain images were collected using a 3D bSSFP sequence with $\alpha=30^{\circ}$, TR/TE $=$ $8.08 / 4.04 \mathrm{~ms}$, field-of-view $(\mathrm{FOV})=218 \times 218 \times 218 \mathrm{~mm}^{3}$, $0.85-\mathrm{mm}$ isotropic resolution, $\mathrm{A} / \mathrm{P}$ and $\mathrm{R} / \mathrm{L}$ phase-encoding, $\mathrm{N}=8$ with $\Delta \phi$ equispaced in the range [0 $2 \pi)$, a 12-channel coil (hardware compressed to 4 channels), and 8 min $50 \mathrm{~s}$ per acquisition. Second, $\mathrm{T}_{2}$-weighted brain images were collected using a 3D turbo spin-echo SPACE (Sampling Perfection with Application optimized Contrasts using different flip angle Evolution) sequence with $\alpha=90-170^{\circ}$, TR $=3000 \mathrm{~ms}$, $\mathrm{TE}=145,257$ and $320 \mathrm{~ms}, \mathrm{FOV}=256 \times 192 \times 224 \mathrm{~mm}^{3}$, $1-\mathrm{mm}$ isotropic resolution, $\mathrm{A} / \mathrm{P}$ and $\mathrm{R} / \mathrm{L}$ phase-encoding, a 32-channel coil, and $11 \mathrm{~min} 12 \mathrm{~s}$ per acquisition. SPACE enables high-spatial-resolution 3D acquisitions for $\mathrm{T}_{2}$-weighted imaging by enabling prolonged echo trains compared to conventional spin-echo sequences [58]. Protocols were approved by the local ethics committee, and informed consent was obtained.

In vivo acquisitions were undersampled retrospectively in two phase-encode dimensions using random and segregated $(\mu=0)$ sampling. For bSSFP datasets, $\mathrm{N}=2,4,6$ and 8 , and $\mathrm{R}=\mathrm{N}$ were used. For $\mathrm{T}_{2}$-weighted datasets, $\mathrm{N}=3$ and $\mathrm{R}=3,4$ and 6 were used.

Profile encoding (PE), parallel imaging (PI), and joint reconstructions were performed on undersampled data. For PE
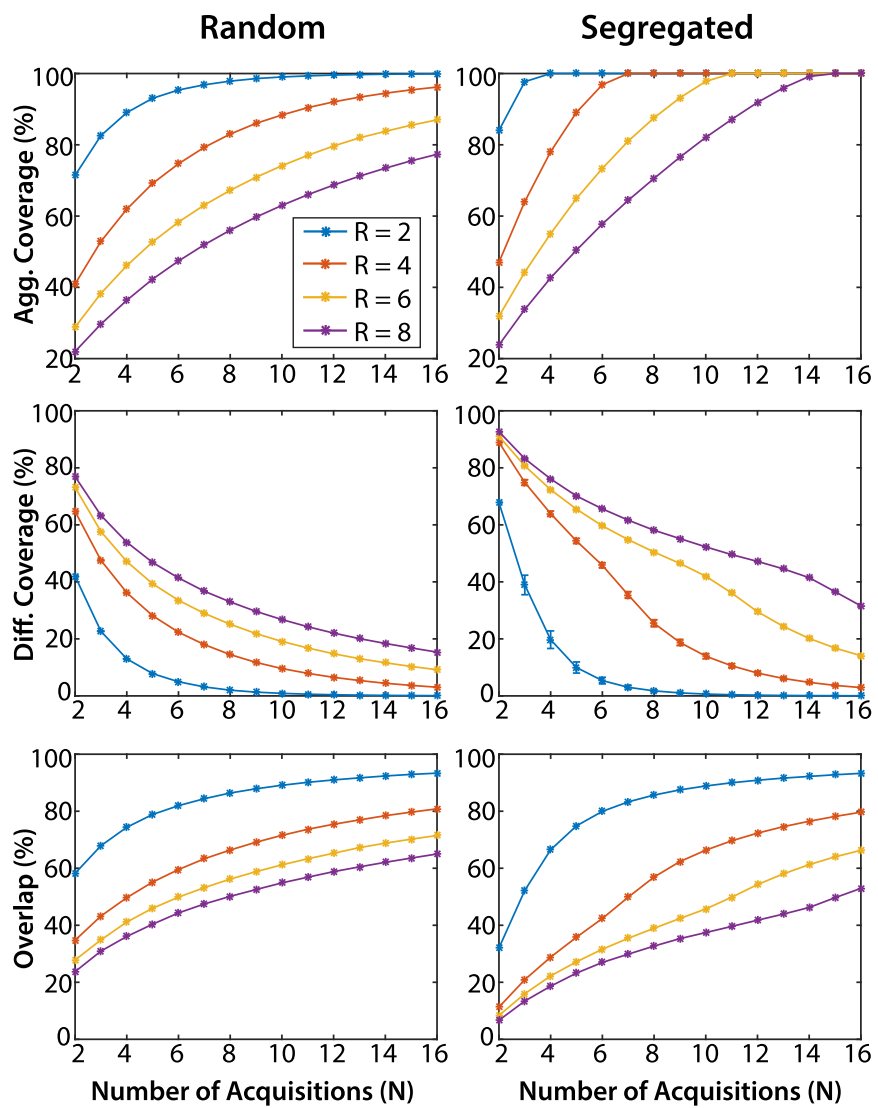

Fig. 3. To assess sampling performance, patterns were generated via random (left column) and segregated sampling (right column, $\mu=0$ ) for $\mathrm{N}=[216]$ and $\mathrm{R}=$ [2 8] on a $256 \times 256$ sampling grid. Aggregate coverage: while random sampling leaves $10-30 \%$ of $k$-space uncovered even at $\mathrm{N}=16$, segregated sampling achieves full coverage within $\mathrm{N}=2 \times \mathrm{R}$ acquisitions. Differential coverage: compared to random sampling, segregated sampling significantly expands the portion of k-space uniquely covered by each pattern. Error bars show mean \pm std. of differential coverage across $\mathrm{N}$ patterns. Percentage overlap: segregated sampling yields reduced overlap, particularly for higher $\mathrm{R}$ and lower $\mathrm{N}$.

and PI, reconstructions were p-norm combined across acquisitions $(p=2)$. For joint reconstructions, images were $p$-norm combined across both coils and acquisitions $(p=2)$. Each image was normalized by mapping the $\% 98$ percentile of pixel intensity to 1 . Reconstruction quality was assessed by PSNR, SSIM and RMSE between the reconstructed images and reference images obtained via fully-sampled Fourier reconstruction. A separate reference image was computed for each $\mathrm{N}_{c}$ by combining across $\mathrm{N}_{c}$ virtual coils. Error and structural similarity maps were also generated for visualization. This procedure was repeated across 5 different cross sections with different instances of sampling patterns.

\section{Results}

\section{A. Simulation Analyses}

Sampling methods were first compared in terms of their aggregate coverage, differential coverage and overlap. Representative patterns from random and segregated sampling are shown in Fig. 2. Measurements for $N=\left[\begin{array}{ll}2 & 16\end{array}\right]$ and $R=\left[\begin{array}{ll}2 & 8\end{array}\right]$ are plotted in Fig. 3 and summarized in Table I. While random sampling can leave $10-30 \%$ of $\mathrm{k}$-space uncovered even at 
TABLE I

Sampling Performance: Segregated Versus Random

\begin{tabular}{|c|c|c|c|c|c|c|c|}
\hline & $\mathbf{N}=\mathbf{2}$ & $\mathbf{N}=\mathbf{3}$ & $N=4$ & $N=6$ & $\mathbf{N}=\mathbf{8}$ & $\mathbf{N}=\mathbf{1 0}$ \\
\hline \multirow{3}{*}{$\mathbf{R}=2$} & Agg. & 12.7 & 15.1 & 10.9 & 4.5 & 2.0 & 1.0 \\
\hline & Diff. & 25.4 & 16.1 & 6.4 & 0.4 & -0.3 & -0.2 \\
\hline & Over. & -25.4 & -15.1 & -7.1 & -2.0 & -0.7 & -0.2 \\
\hline \multirow{3}{*}{$R=4$} & Agg. & 5.9 & 11.3 & 15.9 & 22.3 & 17.1 & 11.7 \\
\hline & Diff. & 23.2 & 27.1 & 27.2 & 23.4 & 10.8 & 4.2 \\
\hline & Over. & -22.7 & -22.0 & -20.5 & -16.7 & -9.1 & -4.8 \\
\hline \multirow{3}{*}{$R=6$} & Agg. & 2.9 & 6.1 & 9.1 & 15.1 & 20.4 & 23.9 \\
\hline & Diff. & 18.1 & 22.7 & 24.5 & 25.9 & 25.5 & 23.0 \\
\hline & Over. & -18.6 & -18.7 & -18.3 & -18.0 & -17.3 & -15.9 \\
\hline \multirow{3}{*}{$R=8$} & Agg. & 2.0 & 4.3 & 6.2 & 10.7 & 14.9 & 19.1 \\
\hline & Diff. & 16.5 & 21.1 & 22.6 & 24.3 & 25.4 & 25.5 \\
\hline & Over. & -16.7 & -17.1 & -16.7 & -16.3 & -16.7 & -16.6 \\
\hline
\end{tabular}

The aggregate coverage (Agg.), differential coverage (Diff.) and overlap (Over.) metrics were calculated for random and segregated sampling $(\mu=0)$ on a $256 \times 256$ grid. Differences in each metric between segregated versus random patterns are listed for $\mathrm{N}=2,3,4,6,8,10$ and $\mathrm{R}=2,4,6,8$.

a

\section{Reconstructions}
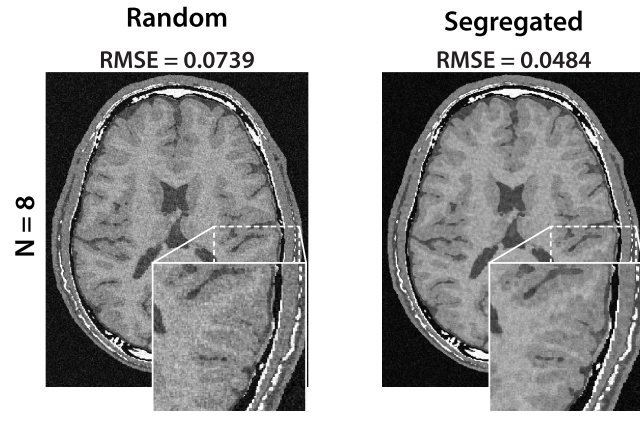

b Error Maps

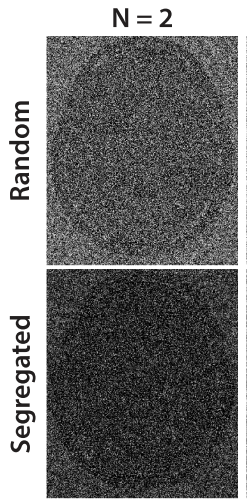

$\mathrm{N}=4$

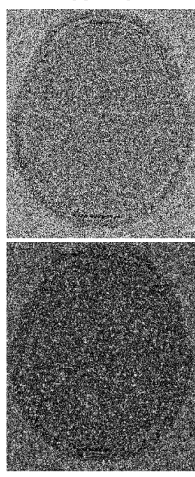

$-35 \mathrm{~dB}$
$\mathrm{N}=6$

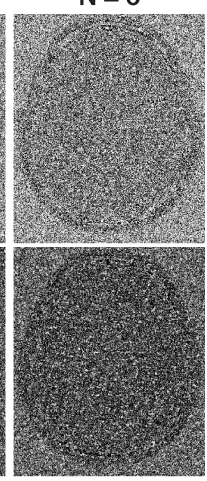

$\mathbf{N}=\mathbf{8}$

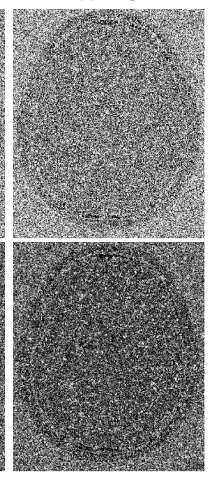

$-20 \mathrm{~dB}$

Fig. 4. Multiple $T_{1}$-weighted numerical phantom images were obtained by undersampling the same data by $\mathrm{N}$ separate patterns. Zerofilled Fourier (ZF) reconstructions were summed across acquisitions. (a) Images with random and segregated sampling $(\mu=0)$ at $\mathrm{N}=8$. Zoomed-in portions are shown in small display windows. Segregated sampling substantially reduces aliasing interference. (b) Error between ZF reconstructions and a fully-sampled reference image is shown in logarithmic scale (see colorbar) for $\mathrm{N}=[2$ 8]. At all $\mathrm{N}$, segregated sampling reduces reconstruction error across the FOV compared to random sampling.

$\mathrm{N}=16$, segregated sampling achieves full coverage within $\mathrm{N}=2 \times \mathrm{R}$ acquisitions. This increased coverage is accompanied by expanded differential coverage within individual patterns and reduced overlap across patterns, particularly a

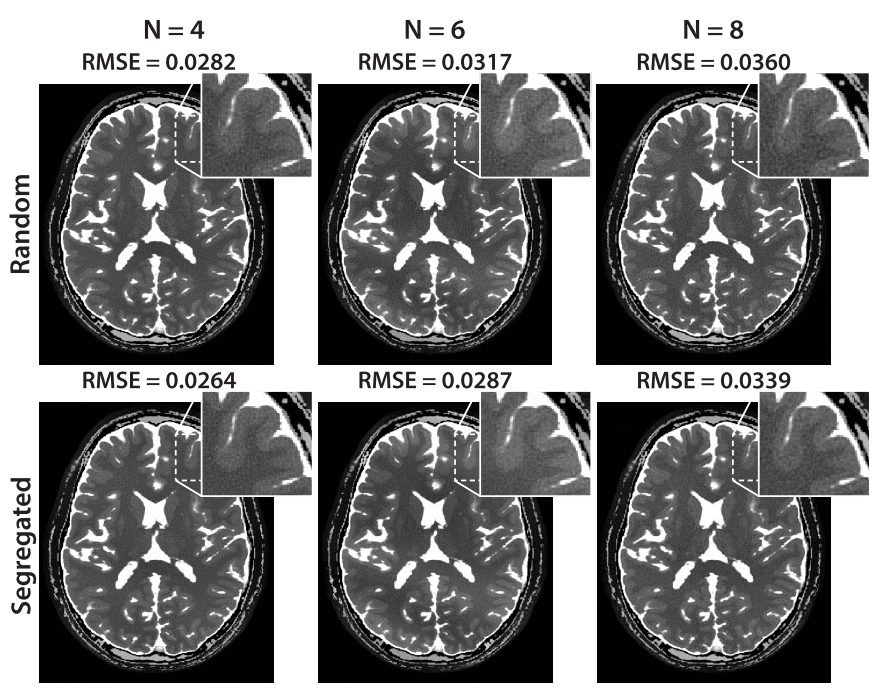

b

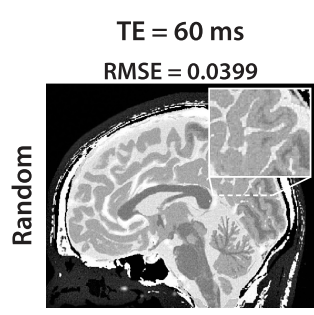

RMSE $=0.0328$

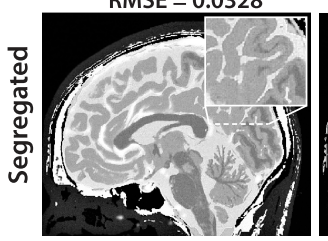

$\mathrm{T}_{2}$-Weighted

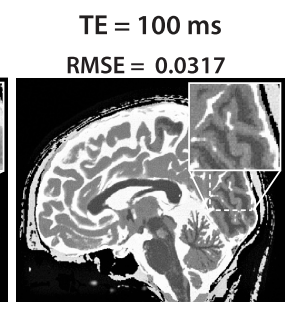

$\mathrm{TE}=140 \mathrm{~ms}$

RMSE $=0.0376$

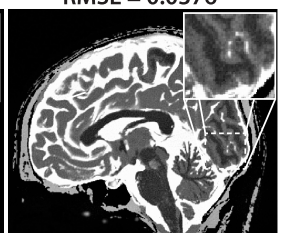

RMSE $=0.0228$

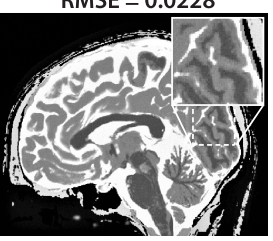

RMSE $=0.0314$

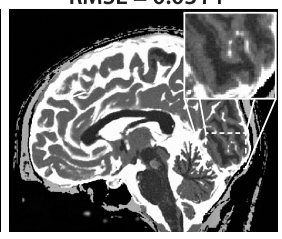

Fig. 5. Numerical brain phantoms were undersampled with random and segregated sampling $(\mu=0)$, and profile-encoding reconstructions were performed. (a) Phase-cycled bSSFP images for $\mathrm{N}=4,6,8$ and $\mathrm{R}=\mathrm{N}$. (b) $\mathrm{T}_{2}$-weighted images for $\mathrm{TE}=60,100$ and $140 \mathrm{~ms}$ and $\mathrm{R}=3$. For both bSSFP and $\mathrm{T}_{2}$-weighted images, reconstructions following segregated sampling have reduced interference from residual aliasing and noise compared to random sampling.

for higher $\mathrm{R}$ and lower N. Segregated sampling achieves $14.6 \pm 0.6 \%$ (mean \pm sem across $\mathrm{N}$, where $\mathrm{R}=\mathrm{N}$ ) higher aggregate coverage, $26.0 \pm 0.4 \%$ higher differential coverage, and $20.2 \pm 1.6 \%$ reduced overlap relative to random sampling. To examine the effect of $\mathrm{k}$-space size, measurements were repeated based on $64 \times 64,128 \times 128,256 \times 256,384 \times 384$ sampling grids (see Supp. Fig. 2 and Supp. Table II). Nearly identical results were obtained in terms of aggregate coverage, differential coverage and overlap independent of $\mathrm{k}$-space size.

To demonstrate segregated sampling, repeated $\mathrm{T}_{1}$-weighted acquisitions of a numerical brain phantom were first simulated. ZF reconstructions are shown in Fig. 4, and PSNR, SSIM and RMSE measurements are listed in Table II for $\mathrm{N}=\left[\begin{array}{ll}2 & 8\end{array}\right]$. Segregated sampling reduces aliasing interference compared to alternative methods, with $3.8 \pm 0.1 \mathrm{~dB}$ higher PSNR and $12.2 \pm 0.5 \%$ higher SSIM than random sampling. 
TABLE II

RECONSTRUCTIONS OF PHANTOM IMAGES

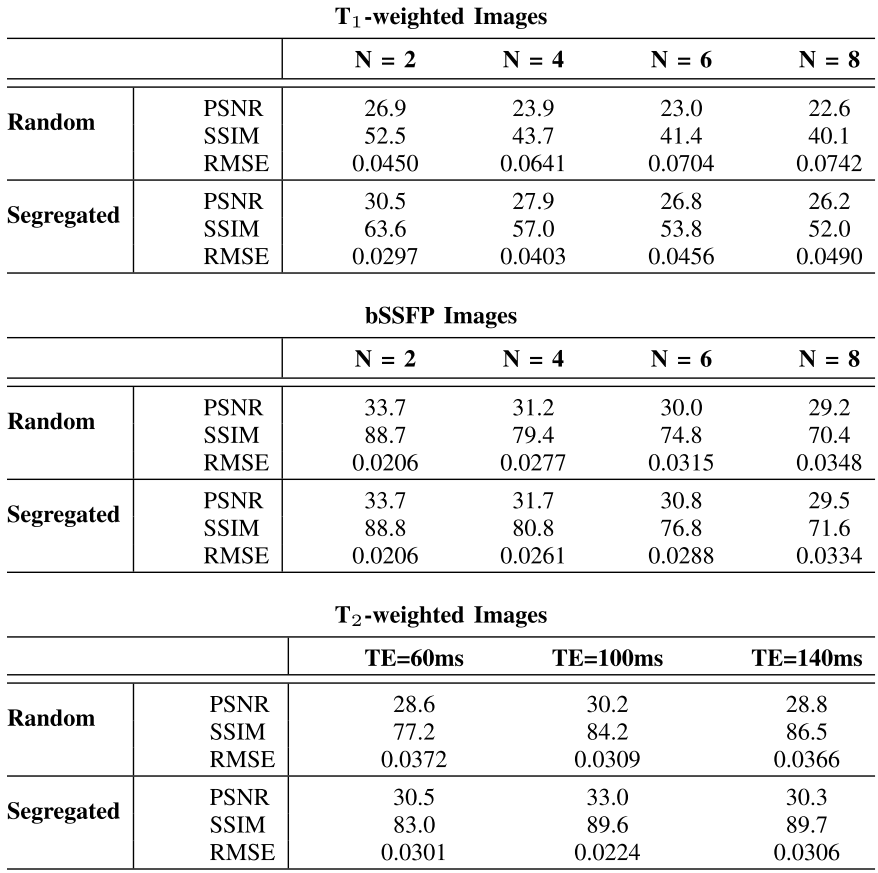

PSNR (dB), SSIM (\%) and RMSE measurements on $\mathrm{T}_{1}$-weighted (top), bSSFP (middle) and $\mathrm{T}_{2}$-weighted (bottom) images of the numerical brain phantom. Random and segregated sampling $(\mu=0)$ were performed at $\mathrm{N}=[28]$ for $\mathrm{T}_{1}$-weighted and bSSFP images, and at $\mathrm{N}=3$ (three echo times) for $\mathrm{T}_{2}$-weighted images. All metrics are reported as mean across 10 different cross sections.

TABLE III

PE RECONSTRUCTIONS Of IN Vivo IMAges

\begin{tabular}{l|c|cccc}
\multicolumn{7}{c}{ bSSFP Images } \\
\hline \hline \multirow{3}{*}{ Random } & & $\mathbf{N}=\mathbf{2}$ & $\mathbf{N}=\mathbf{4}$ & $\mathbf{N}=\mathbf{6}$ & $\mathbf{N = \mathbf { 8 }}$ \\
& PSNR & 34.1 & 28.4 & 27.1 & 26.1 \\
& SSIM & 91.7 & 65.7 & 54.7 & 46.8 \\
& RMSE & 0.0198 & 0.0381 & 0.0442 & 0.0493 \\
\hline \multirow{4}{*}{ Segregated } & PSNR & 34.9 & 29.9 & 28.7 & 27.3 \\
& SSIM & 92.7 & 67.4 & 57.2 & 48.9 \\
& RMSE & 0.0180 & 0.0318 & 0.0367 & 0.0430 \\
\hline
\end{tabular}

\begin{tabular}{l|c|ccc}
\multicolumn{5}{c}{ T $_{2}$-weighted Images } \\
\hline \multirow{3}{*}{ Random } & & $\mathbf{R}=\mathbf{3}$ & $\mathbf{R}=\mathbf{4}$ & $\mathbf{R}=\mathbf{6}$ \\
\hline \hline \multirow{3}{*}{ Segregated } & PSNR & 28.6 & 26.9 & 24.8 \\
& SSIM & 79.6 & 75.5 & 70.4 \\
& RMSE & 0.0370 & 0.0453 & 0.0577 \\
\hline & PSNR & 29.4 & 27.7 & 25.3 \\
& SSIM & 81.0 & 77.0 & 72.0 \\
& RMSE & 0.0339 & 0.0413 & 0.0544 \\
\hline
\end{tabular}

PSNR, SSIM and RMSE measurements on in vivo bSSFP (upper table) and $\mathrm{T}_{2}$ weighted (lower table) images of the brain. Random and segregated $(\mu=0)$ sampling were performed at $\mathrm{N}=2,4,6,8$ with $\mathrm{R}=\mathrm{N}$ for bSSFP images, and at $\mathrm{N}=3$ (three echo times) with $\mathrm{R}=3,4,6$ for $\mathrm{T}_{2}$-weighted images. Profile encoding (PE) reconstructions were computed. Metrics are reported as mean across 5 cross sections for bSSFP data and as mean across 5 cross sections and 3 echo times for $T_{2}$-weighted data.

Following this demonstration on a linear reconstruction, segregated sampling was evaluated based on PE reconstructions of bSSFP acquisitions. Reconstructions of the numerical brain phantom are shown in Fig. 5a. PSNR, SSIM and RMSE on combined bSSFP images are listed in Table II for $\mathrm{N}=\left[\begin{array}{l}2 \\ 2\end{array}\right]$. a

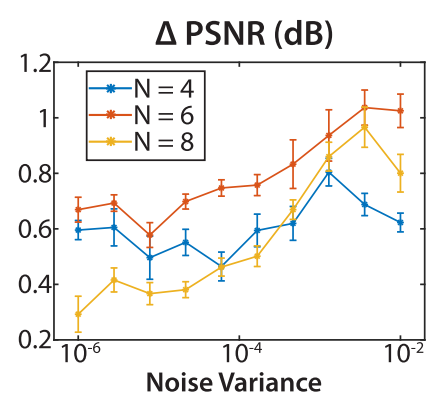

bSSFP

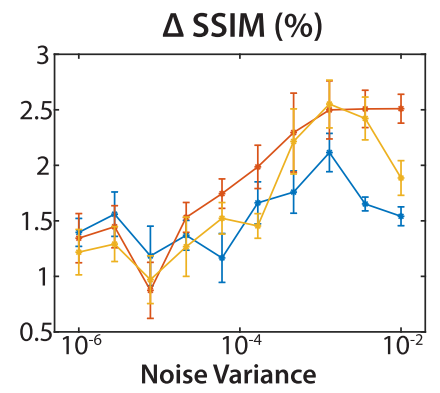

b

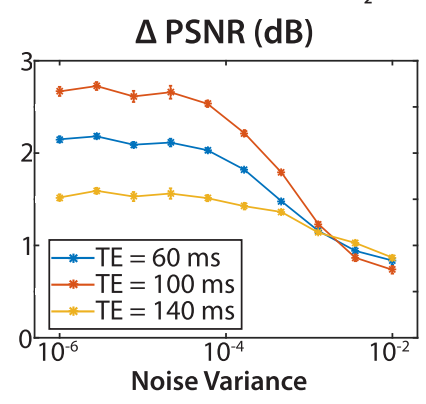

$\mathrm{T}_{2}$-Weighted

C
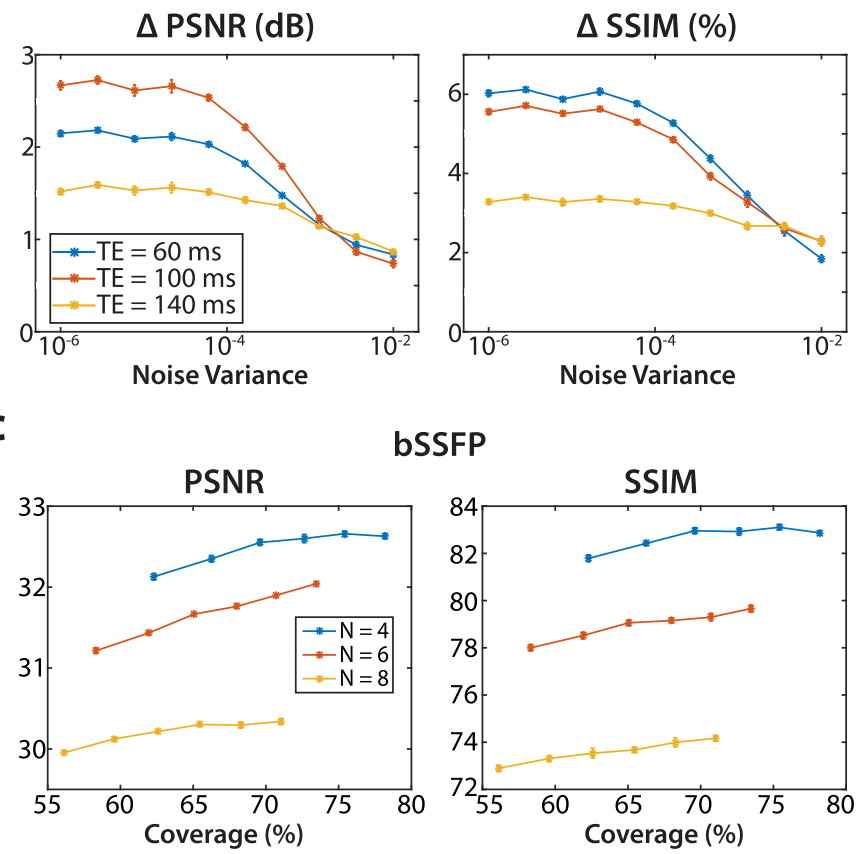

bSSFP

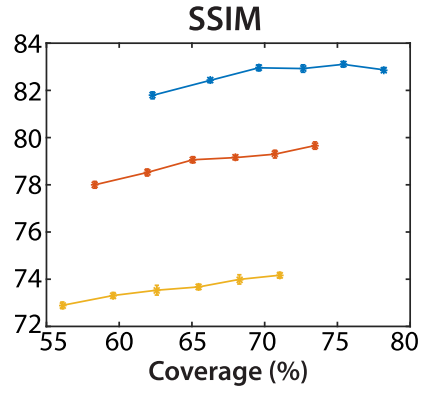

Fig. 6. The effect of noise level and aggregate coverage on sampling performance was examined on numerical brain phantom images. Bivariate Gaussian noise with zero mean and variance in $\left[10^{-6} 10^{-2}\right]$ was added, and $\mathrm{PE}$ reconstructions were performed on noisy data undersampled with random and segregated $(\mu=0)$ patterns $(\mathrm{N}=\mathrm{R})$. Improvements in PSNR and SSIM with segregated sampling over random sampling with respect to noise level are shown: (a) for bSSFP images at $\mathrm{N}=(4$, 6,8 ); (b) for $T_{2}$-weighted images at three echo times (TE). Error bars display mean \pm std across 10 independent sets of noise instance and sampling patterns. Segregated sampling achieves superior performance for a broad range of noise levels. (c) The parameter $\mu$ in Eq. 17 was tuned to systematically vary aggregate k-space coverage from that of random sampling to that of segregated sampling. Phantom bSSFP datasets were undersampled for $\mu=(0,0.2,0.4,0.6,0.8,1.0)$ and $\mathrm{PE}$ reconstructions were obtained for $\mathrm{N}=4,6,8$ and $\mathrm{R}=\mathrm{N}$. Error bars display mean \pm std across 10 independent sets of sampling patterns. At all N, reconstruction quality improves persistently as the aggregate coverage is broadened.

Segregated sampling achieves $0.4 \pm 0.2 \mathrm{~dB}$ higher PSNR and $1.2 \pm 0.4 \%$ higher SSIM compared to random sampling (though they perform similarly at $\mathrm{N}=2$ ). Next, multi-contrast $\mathrm{T}_{2}$-weighted acquisitions of the numerical brain phantom were examined. Reconstructions at three echo times (TE) are shown in Fig. 5b, and PSNR and SSIM for $\mathrm{N}=3$ are listed in Table II. Segregated sampling improves PSNR by $2.1 \pm 0.4 \mathrm{~dB}$ (across TE) and SSIM by $4.8 \pm 0.8 \%$ over random sampling. Similar improvements are obtained for segregated sampling 


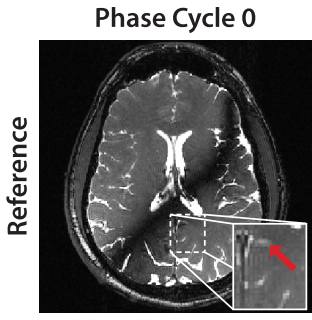

$\mathrm{N}=4$

RMSE $=0.0388$

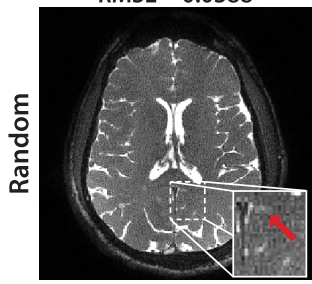

RMSE $=0.0320$
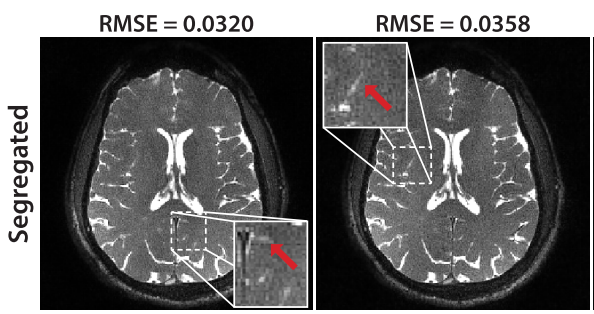

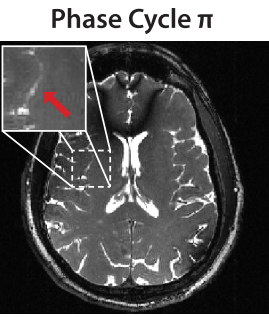

$\mathbf{N}=6$ RMSE $=0.0428$
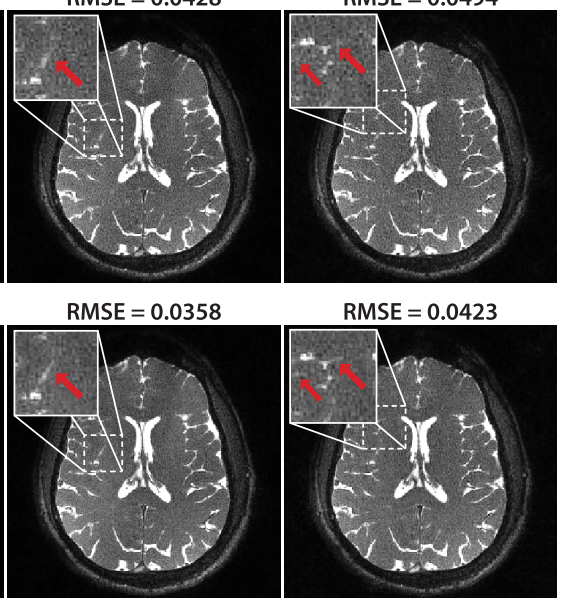

Fig. 7. In vivo bSSFP acquisitions of the brain were undersampled with random and segregated sampling $(\mu=0)$. PE reconstructions were performed for $\mathrm{N}=4,6,8$ and $\mathrm{R}=\mathrm{N}$. Fully-sampled reference images are shown for two individual phase cycles along with the combination image (top row). Reconstructions from random (middle row) and segregated (bottom row) sampling are shown for $N=4,6$, 8 . Segregated sampling provides reduced reconstruction error compared to random sampling. Zoomed-in display windows show detailed features that are poorly depicted with random sampling. These features are sensitively recovered with segregated sampling (see arrows).

over random sampling for the reconstructions of numerical phantoms at lower spatial resolution (see Supp. Fig. 3).

To assess reliability against noise, reconstruction quality was evaluated across a broad range of noise levels. Fig. 6a-b display the difference in PSNR and SSIM between segregated and random sampling. For both bSSFP and $\mathrm{T}_{2}$-weighted images, segregated sampling improves image quality across the entire noise range. The improvements grow for higher noise levels in bSSFP images, where quality metrics were calculated on combined images that average data across acquisitions. In contrast, metrics were calculated on each $\mathrm{T}_{2}$-weighted image without averaging, thus the relatively larger improvements in this case decline with higher noise.

Lastly, the effect of aggregate coverage on sampling performance was examined. Phase-cycled bSSFP acquisitions of the numerical brain phantom were undersampled for varying values of $\mu$, which controls the aggregate coverage. PSNR and SSIM are plotted as a function of aggregate coverage in Fig. 6c. At all N, PSNR and SSIM improve consistently with increased aggregate coverage. Taken together, these results suggest that segregated sampling captures greater information about tissue structure and leads to improved CS recovery due to its expanded coverage and reduced pattern overlap.
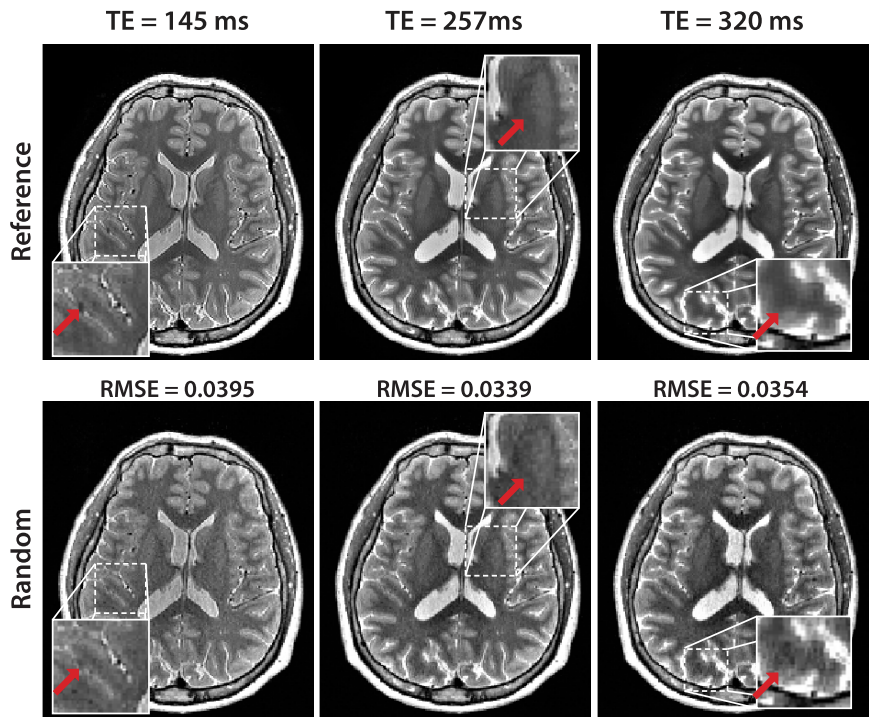

RMSE $=0.0354$

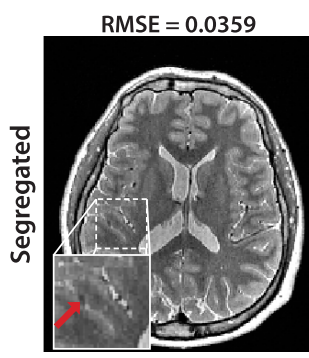

RMSE $=0.0308$
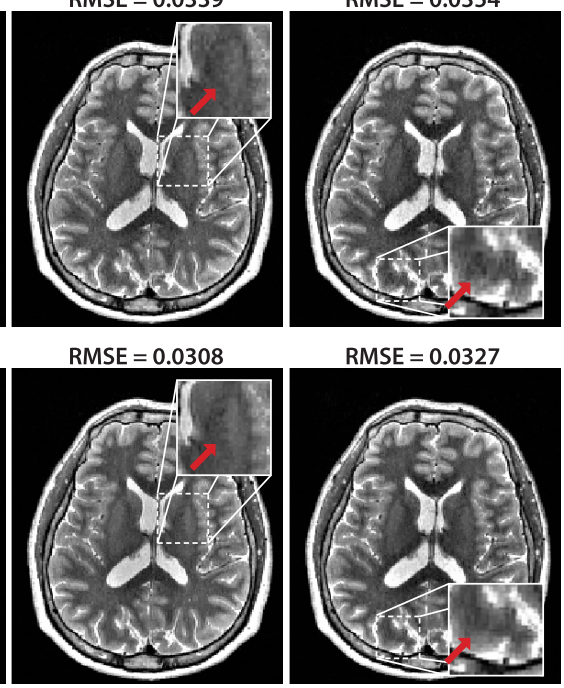

RMSE $=0.0327$

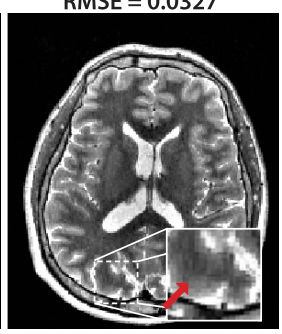

Fig. 8. In vivo $\mathrm{T}_{2}$-weighted acquisitions (at $\mathrm{TE}=145,257$ and $320 \mathrm{~ms}$ ) of the brain were undersampled with random and segregated $(\mu=0)$ sampling. PE reconstructions were performed for $\mathrm{N}=3$ and $\mathrm{R}=\mathrm{N}$. Fully-sampled reference images (top row), reconstructions from random sampling (middle row) and reconstructions from segregated sampling (bottom row) are shown for each TE. Segregated sampling enables improved reconstructions due to reduced aliasing and noise interference. Zoomed-in display windows show limited-contrast or small features that are relatively more visible with segregated sampling (see arrows).

\section{B. In Vivo Analyses}

The proposed strategy was demonstrated on in vivo bSSFP images of the brain. Random and segregated sampling were compared in terms of the respective PE reconstructions combined across acquisitions. Representative reconstructions for $\mathrm{N}=\left[\begin{array}{ll}4 & 8\end{array}\right]$ are shown in Fig. 7 (see Supp. Fig. 4 for respective error and structural similarity maps). Segregated sampling reduces residual errors in bSSFP images. Furthermore, some detailed features that are poorly depicted with random sampling are sensitively recovered with segregated sampling. These observations are supported by PSNR and SSIM measurements listed in Table III. Segregated sampling yields $1.3 \pm 0.2 \mathrm{~dB}$ (across $\mathrm{N}$ ) higher PSNR and $1.8 \pm 0.3 \%$ higher SSIM than random sampling.

Next, in vivo multi-contrast $T_{2}$-weighted images of the brain were considered. PE reconstructions at $\mathrm{N}=3$ displayed in Fig. 8 demonstrate improved quality with segregated sampling (see Supp. Fig. 5 for respective error and structural similarity maps). Several limited-contrast or small features are relatively more visible with segregated sampling. PSNR, SSIM and RMSE measurements are listed in Table III. Segregated sampling yields $0.7 \pm 0.1 \mathrm{~dB}$ (across TE and R) higher PSNR 

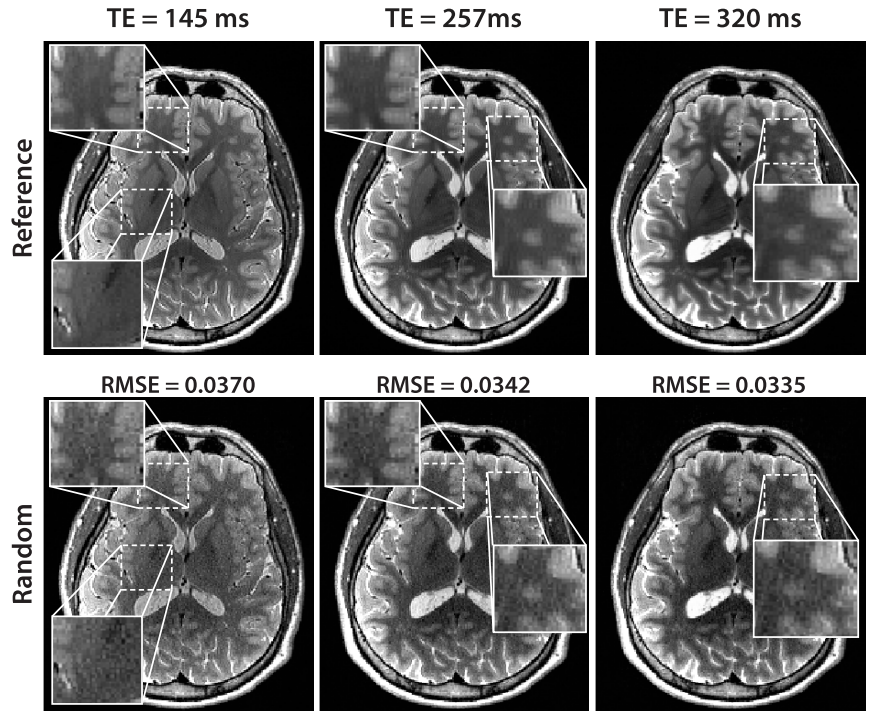

RMSE $=0.0331$

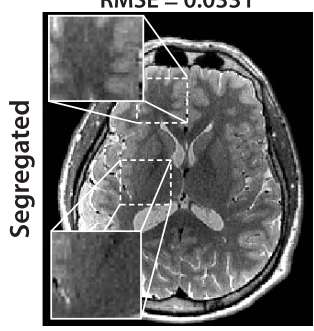

RMSE $=0.0294$

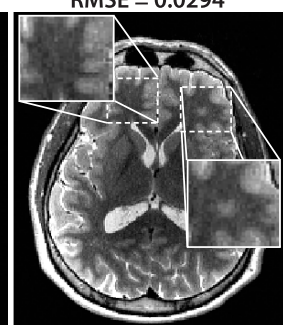

$\mathrm{RMSE}=0.0335$

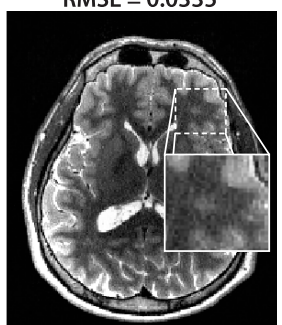

RMSE $=0.0298$

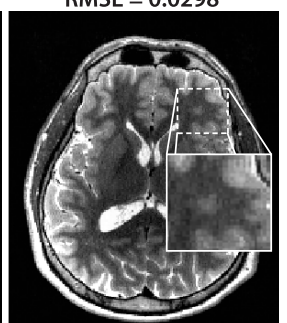

Fig. 9. In vivo $T_{2}$-weighted acquisitions (at $T E=145,257$ and $320 \mathrm{~ms}$ ) of the brain were undersampled with random and segregated $(\mu=0)$ sampling. Joint reconstructions across coils and acquisitions were performed for $\mathrm{N}=3, \mathrm{R}=4$ and $\mathrm{N}_{c}=2$. Fully-sampled reference images (top row), reconstructions from random sampling (middle row) and reconstructions from segregated sampling (bottom row) are shown for each TE. Segregated sampling enables improved reconstructions due to reduced aliasing and noise interference. Zoomed-in display windows show regions where tissue features are relatively more visible with segregated sampling due to enhanced reconstruction quality.

and $1.5 \pm 0.3 \%$ higher SSIM than random sampling (though they perform similarly at $\mathrm{TE}=320 \mathrm{~ms}$ ).

We then examined the utility of segregated sampling for joint reconstruction of multiple-coil multiple-acquisition data. Random and segregated sampling were compared in terms of their joint reconstruction performance on bSSFP and $\mathrm{T}_{2}$-weighted brain images. Representative reconstructions of $\mathrm{T}_{2}$-weighted images at $\mathrm{N}=3, \mathrm{R}=4$ and $\mathrm{N}_{c}=2$ are shown in Fig. 9 (see Supp. Fig. 6 for respective error and structural similarity maps). Similar to PE reconstructions, joint reconstructions visibly benefit from segregated sampling that reduces residual reconstruction errors. PSNR, SSIM and RMSE measurements for bSSFP and $\mathrm{T}_{2}$-weighted images are listed in Table IV. For bSSFP images, segregated sampling yields $0.2 \pm 0.1 \mathrm{~dB}$ (across $\mathrm{N}$ and $\mathrm{N}_{c}$ ) higher PSNR and $0.5 \pm 0.1 \%$ higher SSIM than random sampling. The benefits of segregated sampling are higher for $\mathrm{N}_{c}=2$, where it yields $0.3 \pm 0.1 \mathrm{~dB}$ (across $\mathrm{N}$ ) higher PSNR and $0.7 \pm 0.2 \%$ higher SSIM than random sampling. For $\mathrm{T}_{2}$-weighted images, segregated sampling yields $0.5 \pm 0.1 \mathrm{~dB}$ (across TE, $\mathrm{R}$ and $\mathrm{N}_{c}$ ) higher PSNR and $1.2 \pm 0.2 \%$ higher SSIM than random sampling. The benefits are again higher for $\mathrm{N}_{c}=2$,
TABLE IV

Joint Reconstructions of In Vivo Images

bSSFP Images

\begin{tabular}{|c|c|c|c|c|c|c|}
\hline & & & $\mathbf{N}=\mathbf{2}$ & $N=4$ & $N=6$ & $\mathbf{N}=\mathbf{8}$ \\
\hline \multirow{6}{*}{ छี } & $N$ & PSNR & 34.5 & 31.0 & 29.6 & 26.8 \\
\hline & II & SSIM & 89.2 & 85.8 & 82.3 & 69.9 \\
\hline & Z & RMSE & 0.0189 & 0.0280 & 0.0332 & 0.0456 \\
\hline & + & PSNR & 33.1 & 26.4 & 25.6 & 23.5 \\
\hline & II & SSIM & 88.4 & 67.7 & 68.0 & 68.3 \\
\hline & z & RMSE & 0.0221 & 0.0477 & 0.0526 & 0.0665 \\
\hline \multirow{6}{*}{ 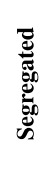 } & $N$ & PSNR & 34.6 & 31.6 & 30.0 & 27.0 \\
\hline & II & SSIM & 89.4 & 86.8 & 83.4 & 70.5 \\
\hline & $\mathbf{Z}$ & RMSE & 0.0186 & 0.0264 & 0.0315 & 0.0445 \\
\hline & $\nabla$ & PSNR & 33.1 & 26.5 & 25.8 & 23.5 \\
\hline & $\|$ & SSIM & 88.5 & 68.1 & 69.0 & 68.2 \\
\hline & Z & RMSE & 0.0221 & 0.0473 & 0.0514 & 0.0667 \\
\hline
\end{tabular}

$\mathbf{T}_{2}$-weighted Images

\begin{tabular}{|c|c|c|c|c|c|}
\hline \multicolumn{6}{|c|}{$\mathbf{T}_{2}$-weighted Images } \\
\hline & & & $\mathbf{R}=\mathbf{3}$ & $R=4$ & $R=6$ \\
\hline \multirow{9}{*}{ 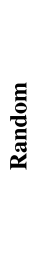 } & 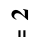 & PSNR & 31.5 & 29.0 & 27.0 \\
\hline & II & SSIM & 88.8 & 83.4 & 77.5 \\
\hline & $\mathbf{z}$ & RMSE & 0.0267 & 0.0354 & 0.0448 \\
\hline & 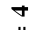 & PSNR & 34.3 & 31.4 & 28.3 \\
\hline & 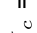 & SSIM & 92.6 & 86.7 & 78.6 \\
\hline & $\mathbf{z}$ & RMSE & 0.193 & 0.0270 & 0.0385 \\
\hline & $\infty$ & PSNR & 34.1 & 29.8 & 25.5 \\
\hline & II & SSIM & 89.9 & 82.4 & 74.7 \\
\hline & $\mathbf{z}$ & RMSE & 0.0197 & 0.0324 & 0.0530 \\
\hline \multirow{9}{*}{ 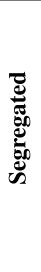 } & $N$ & PSNR & 32.2 & 30.1 & 28.0 \\
\hline & II & SSIM & 90.4 & 85.4 & 79.5 \\
\hline & $\mathbf{z}$ & RMSE & 0.0245 & 0.0314 & 0.0400 \\
\hline & $\nabla$ & PSNR & 34.8 & 31.7 & 28.4 \\
\hline & II & SSIM & 93.3 & 87.1 & 78.9 \\
\hline & $\mathbf{z}$ & RMSE & 0.0183 & 0.0260 & 0.0381 \\
\hline & $\infty$ & PSNR & 34.6 & 30.3 & 25.6 \\
\hline & II & SSIM & 90.9 & 84.0 & 75.3 \\
\hline & $z^{0}$ & RMSE & 0.0188 & 0.0307 & 0.0527 \\
\hline
\end{tabular}

PSNR, SSIM and RMSE measurements on in vivo bSSFP (upper table) and $\mathrm{T}_{2}$ weighted (lower table) images of the brain. Random and segregated $(\mu=0)$ sampling were performed at $\mathrm{R}=\mathrm{N}=2,4,6,8$ for bSSFP images, and at $\mathrm{N}=3$ (three echo times) and $\mathrm{R}=3,4,6$ for $\mathrm{T}_{2}$-weighted images. Joint reconstructions were computed. Metrics are reported as mean across cross sections. For bSSFP data, 5 cross sections were selected in combined images, and for $T_{2}$-weighted data 5 cross sections were selected from images at three echo times.

where segregated sampling yields $0.9 \pm 0.1 \mathrm{~dB}$ (across TE and R) higher PSNR and $1.9 \pm 0.2 \%$ higher SSIM than random sampling. Broader results for joint reconstructions based on uniform-density random, variable-density random and segregated sampling are displayed in Supp. Figs. 7, 8 and listed in Supp. Table III. These results confirm that segregated sampling outperforms both random sampling methods for bSSFP and $\mathrm{T}_{2}$-weighted images.

We also assessed the effect of coil compression rate on the trade-off between the quality and computational efficiency of joint reconstructions. Reconstruction time and performance measurements for $\mathrm{T}_{2}$-weighted images are listed in Supp. Table IV for $\mathrm{R}=\left[\begin{array}{ll}3 & 6\end{array}\right]$ and $\mathrm{N}_{c}=\left[\begin{array}{ll}2 & 16\end{array}\right]$. To enable comparisons among different $\mathrm{N}_{c}$ values in this particular case, the reference image was taken as the combination across all uncompressed coils. While reconstruction time grows exponentially for increasing $\mathrm{N}_{c}$, performance improvements up to $\mathrm{N}_{c}=4$ or 8 (depending on $\mathrm{R}$ ) are followed by modest degradations towards higher $\mathrm{N}_{c}$. These results suggest that decreased estimation fidelity for interpolation kernels at relatively high $\mathrm{N}_{c}$ outweigh the benefits of pooling information 
across a greater number of virtual coils. Thus, intermediate coil compression rates may offer a better compromise between reconstruction time and quality.

Finally, alternative methods for reconstructing multipleacquisition data were evaluated. Specifically, PE and Joint reconstructions based on segregated sampling were compared against PI based on CAIPIRINHA sampling. PI reconstructions were performed based on higher $N_{c}$ than Joint reconstructions to balance the number of information channels available to the two methods. Representative reconstructions of in vivo bSSFP and $\mathrm{T}_{2}$-weighted images are displayed in Supp. Figs. 9 and 10, respectively. PSNR, SSIM and RMSE at various acceleration factors are listed in Supp. Table V. For both bSSFP and $\mathrm{T}_{2}$-weighted images, Joint reconstruction achieves the highest image quality among the three methods, except for bSSFP images at $\mathrm{N}=8$ where Joint and PE perform similarly. For bSSFP images, PE outperforms PI at all $\mathrm{N}$ likely due to relatively lower $\mathrm{N}_{c}$ compared to $\mathrm{N}$. In this case, PI shows suboptimal albeit reasonable performance even when $\mathrm{R}>\mathrm{N}_{c}$. Note that bSSFP reconstructions are obtained after combination across multiple phase-cycled acquisitions. Thus, the PI performance at $\mathrm{R}>\mathrm{N}_{c}$ can be attributed to destructive interference of aliasing artifacts among acquisitions [28]. In contrast, for $\mathrm{T}_{2}$-weighted images, PI yields higher performance than PE likely due to relatively higher $\mathrm{N}_{c}$ compared to $\mathrm{N}$.

\section{Discussion}

CS theory indicates that a system matrix that yields a bounded restricted isometry property (RIP) constant will ensure successful recovery of a sparse signal [34]. Thus, uniform-density random sampling that yields bounded RIP is theoretically motivated for single-acquisition MRI. While system matrices for group-sparse signals remain understudied (but see [59] for an RIP measure for simultaneously acquired, linearly related group-sparse signals), it may be expected that uniform-density random patterns are also adequate for multiple-acquisition MRI assuming perfect recovery is possible. Practical patterns, however, often show deviations from this constraint to ensure reliability against residual aliasing and noise during imperfect recovery (e.g., variable-density patterns in single-acquisition MRI). To improve multiple-acquisition MRI reconstructions, here we proposed statistically segregated patterns containing partly distinct subsets of k-space samples to yield less coherent aliasing artifacts that interfere destructively in the acquisition dimension. This segregation procedure is similar to the minimization of cross-correlation to attain system matrices with lower coherence in CS theory [33]. We empirically demonstrated that segregated sampling lowers reconstruction errors compared to random sampling, although both methods have similar levels of incoherence in the phaseencode dimensions. This finding implies that correlation of patterns in the acquisition dimension is reflective of success in group-sparse recovery, and may motivate future theoretical investigations.

Several previous reports considered sampling strategies for multiple-acquisition data. Earlier work focused on generating incoherent patterns across separate acquisitions [9]. For this purpose, each individual acquisition was accelerated via a distinct random pattern drawn from a common sampling density [14], [25], [27]. Although random sampling theoretically promises successful CS recovery, naive random selection can generate gaps or clusters across the acquisition dimension [40]. In turn, a k-space gap can impair the recovery of unacquired data, whereas a k-space cluster can reduce scan efficiency by collecting redundant information.

To prevent gaps or clusters across temporal frames, recent studies on dynamic MRI incorporated deterministic criteria for sample selection [41]-[43]. With similar motivations, we recently proposed low-correlation sampling to reduce pattern overlap in bSSFP imaging [28]. While low-correlation sampling reduces aliasing artifacts, it uses a search procedure following pattern generation that is suboptimal for minimizing overlap. In contrast, segregated sampling reduces pattern overlap during pattern generation, yielding greater coverage.

Here the enhanced performance of segregated sampling was demonstrated for phase-cycled bSSFP and multi-contrast imaging. The quality improvement in bSSFP images is relatively higher for in vivo datasets compared to simulations (without noise), and the reverse is observed for multi-contrast images. Our analyses also indicate that, at higher noise levels typically encountered in practice, improvements in combined bSSFP images increase whereas those in individual $\mathrm{T}_{2}$-weighted images decrease. Therefore, the observed differences between simulations and in vivo experiments might be attributed to varying noise levels. The precise level of improvement will depend on sequence parameters, tissue structure and experimental conditions. Regardless, segregated sampling is expected to outperform random sampling without imposing any additional computational burden.

A number of avenues can be explored to further improve segregated sampling. Currently, the proposed method optimizes parameters of the polynomial sampling density based on the PSF of the resultant patterns. However, the spectra of MRI images are not guaranteed to strictly follow a power law, and thus a polynomial density may be suboptimal. Previous work suggests that a template of the power spectrum can be used to effectively capture the energy in MRI data [37][39]. Similarly, the initial sampling density in segregated sampling could be designed to match the spectrum estimates for particular anatomies and MRI contrasts.

Another improvement concerns pattern generation based on the chosen sampling density. A stochastic method could result in spatial-frequency gaps or clusters within individual patterns. Our analyses indicate that variable-density random sampling outperforms Poisson-disc sampling in joint reconstructions of bSSFP and $\mathrm{T}_{2}$-weighted acquisitions, hence Poisson sampling was not pursued here. For other datasets or reconstruction methods where Poisson sampling may offer performance benefits, it remains important future work to develop segregated Poisson sampling to increase sampling uniformity [40].

Segregated sampling generates individual patterns sequentially. It is possible that later patterns in the sequence are more constrained in terms of the k-space distribution of sample locations. In this study, we did not observe any degradation 
in the point spread function of the sampling patterns up to $\mathrm{N}=8$. Yet, potential degradations that can arise for larger $\mathrm{N}$ or smaller pattern sizes might be alleviated by implementing more conservative changes in sampling density across acquisitions. Alternatively, k-space can be split into annular segments [60], and the proposed method can be performed on each segment separately. To achieve more balanced sampling, the pattern-generation order for $\mathrm{N}$ acquisitions might be randomized across segments.

Here we demonstrated high-quality $\mathrm{PE}$ and joint reconstructions with acceleration factors up to $\mathrm{R}=8$ in bSSFP and $\mathrm{R}=6$ in $\mathrm{T}_{2}$-weighted acquisitions. Several strategies can be considered to further improve reconstruction quality and maintain higher acceleration factors. First, segregated sampling can be implemented based on CAIPIRINHA-type patterns to lower aliasing energy [61]. Second, SPIRiT reconstructions can be extended to include virtual-coil sensitivities to exploit conjugate symmetry in k-space [62]. A theoretical framework was recently introduced where coil sensitivities are considered to induce varying sampling errors in k-space [63]. This framework could be leveraged to adapt sampling patterns to specific coil arrays, and further optimize sampling performance. A mere increase in the number of acquisitions $(\mathrm{N})$ or coils $\left(\mathrm{N}_{c}\right)$ might also be sufficient. Note, however, that the proposed method segregates patterns across acquisitions but not coils. As such, the benefits of segregated sampling in joint reconstructions can be more modest as $\mathrm{N}_{c}$ increases relative to $\mathrm{N}$.

To conclude, segregated sampling improves multipleacquisition MRI reconstructions by achieving incoherent aliasing both within and across acquisitions. Here we demonstrated improvements for phase-cycled bSSFP and multiple-contrast data compared to conventional sampling methods. Segregated sampling was primarily demonstrated for acceleration in the two-phase encode dimensions of 3D Cartesian acquisitions. Note, however, that adaptation of the proposed method to 1D acceleration in 2D acquisitions is rather straightforward. In principle, the proposed method can also benefit other applications where multiple acquisitions are critical such as peripheral angiography [64] and fat/water separation [47], [65].

\section{References}

[1] I. Koktzoglou and R. R. Edelman, "STAR and STARFIRE for flowdependent and flow-independent noncontrast carotid angiography," Magn. Reson. Med., vol. 61, no. 1, pp. 117-124, Jan. 2009.

[2] N. K. Bangerter et al., "Three-dimensional fluid-suppressed T2-prep flow-independent peripheral angiography using balanced SSFP," Magn. Reson. Imag., vol. 29, no. 8, pp. 1119-1124, Oct. 2011.

[3] S. C. L. Deoni, B. K. Rutt, and T. M. Peters, "Rapid combined $T_{1}$ and $T_{2}$ mapping using gradient recalled acquisition in the steady state," Magn. Reson. Med., vol. 49, no. 3, pp. 515-526, Mar. 2003.

[4] E. U. Saritas, D. Lee, T. Çukur, A. Shankaranarayanan, and D. G. Nishimura, "Hadamard slice encoding for reduced-FOV diffusionweighted imaging," Magn. Reson. Med., vol. 72, no. 5, pp. 1277-1290, 2014.

[5] S. B. Reeder, M. Markl, H. Yu, J. C. Hellinger, R. J. Herfkens, and N. J. Pelc, "Cardiac CINE imaging with IDEAL water-fat separation and steady-state free precession," J. Magn. Reson. Imag., vol. 22, no. 1, pp. 44-52, Jul. 2005.

[6] T. Çukur et al., "Magnetization-prepared IDEAL bSSFP: A flowindependent technique for noncontrast-enhanced peripheral angiography," J. Magn. Reson. Imag., vol. 33, no. 4, pp. 931-939, Apr. 2011.
[7] N. K. Bangerter, B. A. Hargreaves, S. S. Vasanawala, J. M. Pauly, G. E. Gold, and D. G. Nishimura, "Analysis of multiple-acquisition SSFP," Magn. Reson. Med., vol. 51, no. 5, pp. 1038-1047, May 2004.

[8] T. Çukur, M. Lustig, and D. G. Nishimura, "Multiple-profile homogeneous image combination: Application to phase-cycled SSFP and multicoil imaging," Magn. Reson. Med., vol. 60, no. 3, pp. 732-738, Sep. 2008

[9] M. Lustig, D. Donoho, and J. M. Pauly, "Sparse MRI: The application of compressed sensing for rapid MR imaging," Magn. Reson. Med., vol. 58, no. 6, pp. 1182-1195, 2007.

[10] U. Gamper, P. Boesiger, and S. Kozerke, "Compressed sensing in dynamic MRI," Magn. Reson. Med., vol. 59, no. 2, pp. 365-373, Feb. 2008.

[11] M. Uecker, T. Hohage, K. T. Block, and J. Frahm, "Image reconstruction by regularized nonlinear inversion-Joint estimation of coil sensitivities and image content," Magn. Reson. Med., vol. 60, no. 3, pp. 674-682, Sep. 2008.

[12] H. Jung, K. Sung, K. S. Nayak, E. Y. Kim, and J. C. Ye, "k-t FOCUSS: A general compressed sensing framework for high resolution dynamic MRI," Magn. Reson. Med., vol. 61, no. 1, pp. 103-116, 2009.

[13] D. Hernando, P. Kellman, J. P. Haldar, and Z.-P. Liang, "Robust water/fat separation in the presence of large field inhomogeneities using a graph cut algorithm," Magn. Reson. Med., vol. 63, no. 1, pp. 79-90, Jan. 2010.

[14] M. Doneva, P. Börnert, H. Eggers, A. Mertins, J. Pauly, and M. Lustig, "Compressed sensing for chemical shift-based water-fat separation," Magn. Reson. Med., vol. 64, no. 6, pp. 1749-1759, Dec. 2010.

[15] C. N. Wiens, C. M. McCurdy, J. D. Willig-Onwuachi, and C. A. McKenzie, " $\mathrm{R}_{2}^{*}$-corrected water-fat imaging using compressed sensing and parallel imaging," Magn. Reson. Med., vol. 71, no. 2, pp. 608-616, Feb. 2014.

[16] M. Doneva, P. Börnert, H. Eggers, C. Stehning, J. Sénégas, and A. Mertins, "Compressed sensing reconstruction for magnetic resonance parameter mapping," Magn. Reson. Med., vol. 64, no. 4, pp. 1114-1120, Oct. 2010.

[17] B. Zhao, W. Lu, T. K. Hitchens, F. Lam, C. Ho, and Z.-P. Liang, "Accelerated MR parameter mapping with low-rank and sparsity constraints," Magn. Reson. Med., vol. 74, no. 2, pp. 489-498, Aug. 2015.

[18] J. V. Velikina, A. L. Alexander, and A. Samsonov, "Accelerating MR parameter mapping using sparsity-promoting regularization in parametric dimension," Magn. Reson. Med., vol. 70, no. 5, pp. 1263-1273, Nov. 2013.

[19] C. Huang, C. G. Graff, E. W. Clarkson, A. Bilgin, and M. I. Altbach, " $T_{2}$ mapping from highly undersampled data by reconstruction of principal component coefficient maps using compressed sensing," Magn. Reson. Med., vol. 67, no. 5, pp. 1355-1366, May 2012

[20] T. J. Sumpf, M. Uecker, S. Boretius, and J. Frahm, "Model-based nonlinear inverse reconstruction for T2 mapping using highly undersampled spin-echo MRI,' J. Magn. Reson. Imag., vol. 34, no. 2, pp. 420-428, Aug. 2011.

[21] M. I. Menzel et al., "Accelerated diffusion spectrum imaging in the human brain using compressed sensing," Magn. Reson. Med., vol. 66, no. 5, pp. 1226-1233, Nov. 2011.

[22] B. A. Landman, J. A. Bogovic, H. Wan, F. El Zahraa ElShahaby, P.-L. Bazin, and J. L. Prince, "Resolution of crossing fibers with constrained compressed sensing using diffusion tensor MRI," NeuroImage, vol. 59, no. 3, pp. 2175-2186, Feb. 2012.

[23] J. P. Haldar et al., "Improved diffusion imaging through SNR-enhancing joint reconstruction," Magn. Reson. Med., vol. 69, no. 1, pp. 277-289, Jan. 2013.

[24] S. Rapacchi et al., "High spatial and temporal resolution dynamic contrast-enhanced magnetic resonance angiography using compressed sensing with magnitude image subtraction," Magn. Reson. Med., vol. 71, no. 5, pp. 1771-1783, May 2014.

[25] B. Bilgic, V. K. Goyal, and E. Adalsteinsson, "Multi-contrast reconstruction with Bayesian compressed sensing," Magn. Reson. Med., vol. 66, no. 6, pp. 1601-1615, Dec. 2011.

[26] A. Majumdar and R. K. Ward, "Accelerating multi-echo T2 weighted MR imaging: Analysis prior group-sparse optimization," J. Magn. Reson., vol. 210, no. 1, pp. 90-97, May 2011.

[27] J. Huang, C. Chen, and L. Axel, "Fast multi-contrast MRI reconstruction," Magn. Reson. Imag., vol. 32, no. 10, pp. 1344-1352, Dec. 2014

[28] T. Çukur, "Accelerated phase-cycled SSFP imaging with compressed sensing," IEEE Trans. Med. Imag., vol. 34, no. 1, pp. 107-115, Jan. 2015.

[29] G. Puy, P. Vandergheynst, and Y. Wiaux, "On variable density compressive sampling," IEEE Signal Process. Lett., vol. 18, no. 10, pp. 595-598, Oct. 2011 
[30] F. Knoll, K. Bredies, T. Pock, and R. Stollberger, "Second order total generalized variation (TGV) for MRI," Magn. Reson. Med., vol. 65, no. 2, pp. 480-491, Feb. 2011.

[31] M. Murphy, M. Alley, J. Demmel, K. Keutzer, S. Vasanawala, and M. Lustig, "Fast $\ell_{1}$-SPIRiT compressed sensing parallel imaging MRI: Scalable parallel implementation and clinically feasible runtime," IEEE Trans. Med. Imag., vol. 31, no. 6, pp. 1250-1262, Jun. 2012.

[32] D. Liang, B. Liu, J. Wang, and L. Ying, "Accelerating SENSE using compressed sensing," Magn. Reson. Med., vol. 62, no. 6, pp. 1574-1584, Dec. 2009.

[33] D. L. Donoho, M. Elad, and V. N. Temlyakov, "Stable recovery of sparse overcomplete representations in the presence of noise," IEEE Trans. Inf. Theory, vol. 52, no. 1, pp. 6-18, Jan. 2006.

[34] E. Candès and J. Romberg, "Sparsity and incoherence in compressive sampling," Inverse Problems, vol. 23, no. 3, pp. 969-985, Apr. 2007.

[35] S. Hu et al., "3D compressed sensing for highly accelerated hyperpolarized ${ }^{13} \mathrm{C}$ MRSI with in vivo applications to transgenic mouse models of cancer," Magn. Reson. Med., vol. 63, no. 2, pp. 312-321, Feb. 2010.

[36] T. Çukur, M. Lustig, E. U. Saritas, and D. G. Nishimura, "Signal compensation and compressed sensing for magnetization-prepared MR angiography," IEEE Trans. Med. Imag., vol. 30, no. 5, pp. 1017-1027, May 2011.

[37] F. Knoll, C. Clason, C. Diwoky, and R. Stollberger, "Adapted random sampling patterns for accelerated MRI," Magn. Reson. Mater. Phys. Biol. Med., vol. 24, no. 1, pp. 43-50, Jan. 2011.

[38] D. Liang, E. V. R. DiBella, R.-R. Chen, and L. Ying, " $k-t$ ISD Dynamic cardiac MR imaging using compressed sensing with iterative support detection," Magn. Reson. Med., vol. 68, no. 1, pp. 41-53, Jul. 2012.

[39] R. Raja and N. Sinha, "Adaptive k-space sampling design for edgeenhanced DCE-MRI using compressed sensing," Magn. Reson. Imag., vol. 32, no. 7, pp. 899-912, Sep. 2014.

[40] M. Lustig and J. M. Pauly, "SPIRiT: Iterative self-consistent parallel imaging reconstruction from arbitrary k-space," Magn. Reson. Med., vol. 64, no. 2, pp. 457-471, Aug. 2010.

[41] R. Ahmad, H. Xue, S. Giri, Y. Ding, J. Craft, and O. P. Simonetti, "Variable density incoherent spatiotemporal acquisition (VISTA) for highly accelerated cardiac MRI," Magn. Reson. Med., vol. 74, no. 5, pp. 1266-1278, Nov. 2015.

[42] W. Kim, Y. Zhou, J. Lyu, and L. Ying, "Conflict-cost based random sampling design for parallel MRI with low rank constraints," Proc. SPIE, vol. 9484, pp. 94840P-1-94840P-10, May 2015.

[43] E. Levine, B. Daniel, S. Vasanawala, B. Hargreaves, and M. Saranathan, "3D Cartesian MRI with compressed sensing and variable view sharing using complementary poisson-disc sampling," Magn. Reson. Med. vol. 77, no. 5, pp. 1774-1785, Apr. 2016.

[44] J. I. Tamir et al., " $T_{2}$ shuffling: Sharp, multicontrast, volumetric fast spin-echo imaging," Magn. Reson. Med., vol. 77, no. 1, pp. 180-195, 2017.

[45] K. Sung and B. A. Hargreaves, "High-frequency subband compressed sensing MRI using quadruplet sampling," Magn. Reson. Med., vol. 70, no. 5, pp. 1306-1318, Nov. 2013.

[46] T. Çukur, N. K. Bangerter, and D. G. Nishimura, "Enhanced spectral shaping in steady-state free precession imaging," Magn. Reson. Med., vol. 58, pp. 1216-1223, Dec. 2007.
[47] B. Quist, B. A. Hargreaves, T. Çukur, G. R. Morrell, G. E. Gold, and N. K. Bangerter, "Simultaneous fat suppression and band reduction with large-angle multiple-acquisition balanced steady-state free precession," Magn. Reson. Med., vol. 67, no. 4, pp. 1004-1012, Apr. 2012.

[48] E. Ilicak, L. K. Senel, E. Biyik, and T. Çukur, "Profile-encoding reconstruction for multiple-acquisition balanced steady-state free precession imaging," Magn. Reson. Med., vol. 78, no. 4, pp. 1316-1329, Oct. 2017.

[49] G. E. Gold, E. Han, J. Stainsby, G. Wright, J. Brittain, and C. Beaulieu, "Musculoskeletal MRI at 3.0 T: Relaxation times and image contrast," AJR Amer. J. Roentgenol., vol. 183, no. 2, pp. 343-351, Aug. 2004.

[50] M. A. Bernstein, K. King, and X. Zhou, Handbook of MRI Pulse Sequences, 1st ed. Burlington, MA, USA: Elsevier, 2004.

[51] M. Misaki et al., "Contrast enhancement by combining T1- and T2weighted structural brain MR images," Magn. Reson. Med., vol. 74, no. 6, pp. 1609-1620, Dec. 2015.

[52] F. A. Breuer et al., "Controlled aliasing in volumetric parallel imaging (2D CAIPIRINHA)," Magn. Reson. Med., vol. 55, no. 3, pp. 549-556, 2006.

[53] M. Bydder, D. J. Larkman, and J. V. Hajnal, "Combination of signals from array coils using image-based estimation of coil sensitivity profiles," Magn. Reson. Med., vol. 47, no. 3, pp. 539-548, 2002.

[54] M. A. Griswold et al., "Generalized autocalibrating partially parallel acquisitions (GRAPPA)," Magn. Reson. Med., vol. 47, no. 6, pp. 1202-1210, 2002.

[55] E. Biyik, E. Ilicak, and T. Çukur, "Reconstruction by calibration over tensors for multi-coil multi-acquisition balanced SSFP imaging," Magn Reson. Med., vol. 79, no. 5, pp. 2542-2554, 2018.

[56] T. Zhang, J. M. Pauly, S. S. Vasanawala, and M. Lustig, "Coil compression for accelerated imaging with Cartesian sampling," Magn. Reson. Med., vol. 69, no. 2, pp. 571-582, Feb. 2013.

[57] G. J. Stanisz et al., " $\mathrm{T}_{1}, \mathrm{~T}_{2}$ relaxation and magnetization transfer in tissue at 3T," Magn. Reson. Med., vol. 54, no. 3, pp. 507-512, Sep. 2005.

[58] R. F. Busse, H. Hariharan, A. Vu, and J. H. Brittain, "Fast spin echo sequences with very long echo trains: Design of variable refocusing flip angle schedules and generation of clinical $T_{2}$ contrast," Magn. Reson. Med., vol. 55, no. 5, pp. 1030-1037, May 2006.

[59] Y. C. Eldar, P. Kuppinger, and H. Bolcskei, "Block-sparse signals: Uncertainty relations and efficient recovery," IEEE Trans. Signal Process., vol. 58, no. 6, pp. 3042-3054, Jun. 2010.

[60] T. Çukur, J. M. Santos, D. G. Nishimura, and J. M. Pauly, "Varying kernel-extent gridding reconstruction for undersampled variable-density spirals," Magn. Reson. Med., vol. 59, no. 1, pp. 196-201, Jan. 2008.

[61] D. Polak et al., "Wave-CAIPI for highly accelerated MP-RAGE imaging," Magn. Reson. Med., vol. 79, no. 1, pp. 401-406, Jan. 2018.

[62] B. Bilgic et al., "Improving parallel imaging by jointly reconstructing multi-contrast data," Magn. Reson. Med., vol. 80, no. 2, pp. 619-632, Aug. 2018.

[63] V. Athalye, M. Lustig, and M. Uecker, "Parallel magnetic resonance imaging as approximation in a reproducing kernel Hilbert space," Inverse Problems, vol. 31, no. 4, p. 045008, 2015.

[64] K. T. Kwon, H. H. Wu, T. Shin, T. Cukur, M. Lustig, and D. G. Nishimura, "Three-dimensional magnetization-prepared imaging using a concentric cylinders trajectory," Magn. Reson. Med., vol. 71, no. 5, pp. 1700-1710, May 2014.

[65] T. Çukur and D. G. Nishimura, "Fat-water separation with alternating repetition time balanced SSFP," Magn. Reson. Med., vol. 60, pp. 479-484, Aug. 2008. 